\title{
\begin{tabular}{l|l} 
Mitraries & DSpace@MIT
\end{tabular}
}

\author{
MIT Open Access Articles
}

\section{IDENTIFYING LUMINOUS ACTIVE GALACTIC NUCLEI IN DEEP SURVEYS: REVISED IRAC SELECTION CRITERIA}

The MIT Faculty has made this article openly available. Please share how this access benefits you. Your story matters.

Citation: Donley, J. L., A. M. Koekemoer, M. Brusa, P. Capak, C. N. Cardamone, F. Civano, 0. Ilbert, et al. “IDENTIFYING LUMINOUS ACTIVE GALACTIC NUCLEI IN DEEP SURVEYS: REVISED IRAC SELECTION CRITERIA.” The Astrophysical Journal 748, no. 2 (March 16, 2012): 142. (C) 2012 The American Astronomical Society

As Published: http://dx.doi.org/10.1088/0004-637x/748/2/142

Publisher: IOP Publishing

Persistent URL: http://hdl.handle.net/1721.1/95575

Version: Final published version: final published article, as it appeared in a journal, conference proceedings, or other formally published context

Terms of Use: Article is made available in accordance with the publisher's policy and may be subject to US copyright law. Please refer to the publisher's site for terms of use. 


\title{
IDENTIFYING LUMINOUS ACTIVE GALACTIC NUCLEI IN DEEP SURVEYS: REVISED IRAC SELECTION CRITERIA
}

\author{
J. L. Donley ${ }^{1,14}$, A. M. Koekemoer ${ }^{1}$, M. Brusa ${ }^{2}$, P. Capak $^{3}$, C. N. Cardamone ${ }^{4}$, F. Civano ${ }^{5}$, O. Ilbert ${ }^{6}$, C. D. Impey $^{7}$, \\ J. S. Kartaltepe ${ }^{8}$, T. Miyaji ${ }^{9,10}$, M. Salvato ${ }^{2}$, D. B. Sanders ${ }^{11}$, J. R. Trump ${ }^{12}$, and G. Zamorani ${ }^{13}$ \\ ${ }^{1}$ Space Telescope Science Institute, 3700 San Martin Drive, Baltimore, MD 21218, USA; donley@ stsci.edu \\ ${ }^{2}$ Max Planck Institut für extraterrestrische Physik, Giessenbachstrasse 1, D-85748 Garching bei München, Germany \\ ${ }^{3}$ Spitzer Science Center, California Institute of Technology, MC 220-6, 1200 East California Boulevard, Pasadena, CA 91125, USA \\ ${ }^{4}$ Department of Physics, Massachusetts Institute of Technology, 77 Massachusetts Avenue, Cambridge, MA 02139, USA \\ ${ }^{5}$ Harvard Smithsonian Center for Astrophysics, 60 Garden Street, Cambridge, MA 02138, USA \\ ${ }^{6}$ Laboratoire d'Astrophysique de Marseille (UMR 6110), CNRS-Université de Provence, 38 rue Frédéric Joliot-Curie, 13388 Marseille Cedex 13, France \\ ${ }^{7}$ Steward Observatory, University of Arizona, 933 North Cherry Avenue, Tucson, AZ 85721, USA \\ ${ }^{8}$ National Optical Astronomy Observatory, 950 North Cherry Avenue, Tucson, AZ 85719, USA \\ ${ }^{9}$ Instituto de Astronomía, Universidad Nacional Autónoma de México, Ensenada, Baja California, Mexico \\ ${ }^{10}$ Center for Astrophysics and Space Sciences, University of California, San Diego, 9500 Gilman Drive, La Jolla, CA 92093-0424, USA \\ ${ }^{11}$ Institute for Astronomy, University of Hawaii, 2680 Woodlawn Drive, Honolulu, HI 96822, USA \\ ${ }^{12}$ UCO/Lick, University of California, Santa Cruz, CA 95064, USA \\ ${ }^{13}$ INAF-Osservatorio Astronomico di Bologna, via Ranzani 1, I-40127 Bologna, Italy \\ Received 2011 August 4; accepted 2012 January 18; published 2012 March 16
}

\begin{abstract}
Spitzer/IRAC selection is a powerful tool for identifying luminous active galactic nuclei (AGNs). For deep IRAC data, however, the AGN selection wedges currently in use are heavily contaminated by star-forming galaxies, especially at high redshift. Using the large samples of luminous AGNs and high-redshift star-forming galaxies in COSMOS, we redefine the AGN selection criteria for use in deep IRAC surveys. The new IRAC criteria are designed to be both highly complete and reliable, and incorporate the best aspects of the current AGN selection wedges and of infrared power-law selection while excluding high-redshift star-forming galaxies selected via the BzK, distant red galaxy, Lyman-break galaxy, and submillimeter galaxy criteria. At QSO luminosities of $\log L_{2-10 \mathrm{keV}}\left(\mathrm{erg} \mathrm{s}^{-1}\right) \geqslant 44$, the new IRAC criteria recover 75\% of the hard X-ray and IRAC-detected XMM-COSMOS sample, yet only 38\% of the IRAC AGN candidates have X-ray counterparts, a fraction that rises to $52 \%$ in regions with Chandra exposures of 50-160 ks. X-ray stacking of the individually X-ray non-detected AGN candidates leads to a hard $\mathrm{X}$-ray signal indicative of heavily obscured to mildly Compton-thick obscuration $\left(\log N_{\mathrm{H}}\left(\mathrm{cm}^{-2}\right)=23.5 \pm 0.4\right)$. While IRAC selection recovers a substantial fraction of luminous unobscured and obscured AGNs, it is incomplete to low-luminosity and host-dominated AGNs.
\end{abstract}

Key words: galaxies: active - infrared: galaxies - X-rays: galaxies

Online-only material: color figures

\section{INTRODUCTION}

While supermassive black hole (SMBH) growth and galaxy formation were once assumed to proceed independently of one another, a new picture is emerging in which common triggering and/or feedback mechanisms drive the formation and evolution of both SMBHs and their hosts. To determine which processes (e.g., secular evolution or major galaxy mergers) are primarily responsible for moving a galaxy onto the present-day $M-\sigma$ relation, however, we first require a complete census of luminous active galactic nucleus (AGN) activity.

In X-rays, the typical AGN outshines even the most actively star-forming galaxy, and as such, deep X-ray surveys provide the most reliable means of AGN selection. However, while $\mathrm{X}$-rays penetrate low to moderate columns of obscuring dust and gas, 2-10 keV X-ray surveys miss a significant fraction of moderately obscured AGNs $\left(\sim 25 \%\right.$ at $\left.N_{\mathrm{H}}=10^{23} \mathrm{~cm}^{-2}\right)$ and nearly all Compton-thick AGNs $\left(N_{\mathrm{H}}>10^{24} \mathrm{~cm}^{-2}\right.$; Treister et al. 2004; Ballantyne et al. 2006; Tozzi et al. 2006). From fits to the cosmic X-ray background, Gilli et al. (2007) predict that both moderately obscured and Compton-thick AGNs are as numerous as unobscured AGNs at high luminosity $\left(\log L_{0.5-2 \mathrm{keV}}\left(\mathrm{erg} \mathrm{s}^{-1}\right)>43.5\right)$, and are four times as numerous

\footnotetext{
${ }^{14}$ Giacconi Fellow.
}

as unobscured AGNs at low luminosity $\left(\log L_{0.5-2 \mathrm{keV}}\left(\mathrm{erg} \mathrm{s}^{-1}\right)<\right.$ 43.5). The obscured and Compton-thick AGNs missed in deep $\mathrm{X}$-ray surveys therefore serve not only as important probes of $\mathrm{SMBH} /$ galaxy co-evolution, but likely constitute a significant fraction of the total AGN population at all luminosities.

To identify obscured AGNs not recovered by X-ray surveys, studies have turned to the mid-infrared (MIR). Not only does the MIR emission from AGN-heated dust trace the reprocessed radiation absorbed in other wavebands, but it is itself relatively insensitive to intervening obscuration. MIR selection therefore identifies many heavily obscured AGNs, nearly half of which are missed in deep X-ray surveys (Donley et al. 2008). While many MIR-based selection criteria are therefore designed to specifically target heavily obscured AGNs (e.g., Daddi et al. 2007a; Dey et al. 2008; Fiore et al. 2008, 2009), Spitzer/IRAC selection is sensitive to the hot dust signature present in $\gtrsim 80 \%-95 \%$ of luminous AGNs regardless of obscuration (Hao et al. 2010, 2011). As such, IRAC selection is a potentially powerful technique not only for identifying the heavily obscured AGNs missed in the X-ray, but also for selecting luminous obscured and unobscured AGNs when deep X-ray data are unavailable.

The IRAC color-color cuts most commonly used for AGN selection were defined by Lacy et al. (2004, 2007) and Stern et al. (2005) using shallow IRAC data to which additional flux 
cuts at $8 \mu \mathrm{m}, 24 \mu \mathrm{m}$, or $R$ band served to reject all but the brightest sources $\left(S_{8 \mu \mathrm{m}} \geqslant 1 \mathrm{mJy}\right.$, Lacy et al. $2004 ; R<21.5$ and $S_{8.0 \mu \mathrm{m}} \geqslant 76 \mu \mathrm{Jy}$, Stern et al. 2005; $S_{24 \mu \mathrm{m}} \gtrsim 5 \mathrm{mJy}$, Lacy et al. 2007). While these initial color cuts therefore effectively select luminous AGNs in samples containing only AGNs and bright, low-redshift star-forming galaxies (see also Sajina et al. 2005), they extend into regions of IRAC color space populated by moderate- to high-redshift $(z \gtrsim 0.5)$ star-forming galaxies in the deep IRAC surveys now available across many cosmological fields (e.g., Barmby et al. 2006; Donley et al. 2007, 2008; Cardamone et al. 2008; Yun et al. 2008; Brusa et al. 2009; Desai et al. 2009; Richards et al. 2009; Assef et al. 2010; Park et al. 2010). IRAC power-law selection, which identifies only the most robust of the IRAC color-selected AGNs, has therefore been adopted to minimize contamination by normal galaxies (Alonso-Herrero et al. 2006; Donley et al. 2007, 2008). This technique, however, depends on both the IRAC photometry and the often-underestimated photometric errors, adding a degree of complexity not present in simple color-color cuts.

Our understanding of the MIR source population has increased substantially in recent years, thanks in part to the Spitzer/IRS spectra now available for large samples of both local and high-redshift sources, and in part to the availability of deep IRAC data in multi-wavelength survey fields. It is therefore time to revisit the IRAC selection of AGNs and redefine the selection criteria for use in deep survey fields. To do so, we focus on the $2 \mathrm{deg}^{2}$ Cosmic Evolution Survey (COSMOS; Scoville et al. 2007; Koekemoer et al. 2007). Unlike the deeper Great Observatories Origins Deep Survey (GOODS) fields, the combined size and depth of COSMOS provides large samples of both luminous AGNs and normal IRAC-detected star-forming galaxies out to $z \sim 3$. Furthermore, intensive spectroscopic follow-up campaigns and photometric redshifts tuned to both AGNs and normal galaxies give near-complete redshift constraints on both the AGN and star-forming populations (Lilly et al. 2007; Trump et al. 2009; Salvato et al. 2009; Ilbert et al. 2009).

This paper is organized as follows. In Section 2, we provide an overview of IRAC AGN selection and discuss which AGNs should reliably be selected by this method. We present the relevant COSMOS data sets in Section 3, and we discuss in Section 4 the IRAC power-law-selected AGNs in COSMOS. In Section 5, we present the properties of the XMM-COSMOS sample, and in Section 6, we summarize the XMM sample's trends in IRAC color space. We then investigate the properties of the full IRAC sample in Section 7 and of high-redshift star-forming galaxies in Section 8. In Section 9, we present the revised selection criteria, and we summarize the results in Section 10. Throughout the paper, we assume the following cosmology: $\left(\Omega_{\mathrm{m}}, \Omega_{\Lambda}, H_{0}\right)=\left(0.27,0.73,70.5 \mathrm{~km} \mathrm{~s}^{-1} \mathrm{Mpc}^{-1}\right)$, and we quote all magnitudes in the $\mathrm{AB}$ system unless otherwise noted.

\section{IRAC AGN SELECTION: AN OVERVIEW}

The premise behind IRAC AGN selection is illustrated in Figure 1, where we plot composite AGN + starburst spectral energy distributions (SEDs) constructed using the QSO1 and M82 templates of Polletta et al. (2008). The SEDs of normal starforming galaxies display a prominent dip between the $1.6 \mu \mathrm{m}$ stellar bump and the long-wavelength emission from starformation-heated dust $\left(T_{\text {dust }} \sim 25-50 \mathrm{~K}\right)$. Dust near an AGN's central engine, however, can reach a sublimation temperature of $T_{\text {dust }} \sim 1000-1500 \mathrm{~K}$ and thus radiate into the near-infrared
(NIR). If the AGN is sufficiently luminous compared to its host galaxy, the superposition of blackbody emission from the AGN-heated dust will fill in the dip in the galaxy's SED and produce a red, power-law-like thermal continuum across the IRAC bands. As shown in Figure 1, while the UV-optical SEDs of obscured and unobscured AGNs bear little resemblance to one another, this characteristic MIR spectral shape should remain so long as the obscuring medium is optically thin at NIR-MIR wavelengths.

The optical thickness of the obscuring medium at NIR-MIR wavelengths depends on the structure of the torus. A smooth and geometrically thick torus will obscure even the MIR emission from type 2 AGNs, leading to a large offset in MIR luminosity between type 1 and type 2 AGNs (Pier \& Krolik 1993). While no studies have observed the degree of MIR anisotropy predicted by smooth torus models, type 2 AGNs do appear to be approximately three to five times fainter in the MIR than type 1 AGNs when the radio continuum luminosity is used to constrain the AGN's intrinsic luminosity (Heckman 1995; Buchanan et al. 2006; Haas et al. 2008; Leipski et al. 2010). However, no offset in MIR luminosity is observed when the AGN luminosity is instead normalized by the absorption-corrected X-ray luminosity and when the AGN's contribution to the MIR emission is isolated either spatially or spectrally (Lutz et al. 2004; Gandhi et al. 2009). This suggests that the radio-based comparison may be biased, at least in part, by the contribution from beamed synchrotron emission in type 1 AGNs (Cleary et al. 2007).

While the observed degree of MIR anisotropy therefore remains an open question, a low optical depth is a natural consequence of clumpy torus models, which predict only weak NIR-MIR anisotropies that essentially disappear by $\lambda \sim 12 \mu \mathrm{m}$ (e.g., Nenkova et al. 2008a, 2008b). Even at $1 \mu \mathrm{m}$, the clumpy torus model of Nenkova et al. (2008b) predicts an edge-to-pole flux ratio of $\lesssim 5$ for a cloud optical depth of $\tau_{\mathrm{V}}=60$. For samples of type 1 and type 2 AGNs averaged across all viewing angles, we might therefore expect only small systematic offsets in the observed MIR emission, and only at the lowest rest-frame wavelengths probed by the IRAC bands. We will investigate this issue in more detail in Sections 6 and 9.

\subsection{IRAC Colors of AGN/Star-forming Composites}

To illustrate the expected IRAC colors of galaxies with varying AGN contributions, we plot in Figures 2 and 3 the $0<z<3$ IRAC colors of composite SEDs constructed from the QSO1, elliptical (ell2), starburst (M82), and ULIRG (IRAS 22491) templates of Polletta et al. (2008) and the star-forming template of Dale \& Helou (2002), plotted in both Lacy et al. $(2004,2007)$ and Stern et al. (2005) color space (see also Assef et al. 2011). To allow for the effects of reddening, we apply additional extinctions of $A_{\mathrm{V}}=0-2$ and $A_{\mathrm{V}}=0-20$ to the starforming and AGN components, respectively, using the Draine (2003) extinction curve (e.g., Hickox et al. 2007; Hou et al. 2011).

We also show in Figures 2 and 3 the AGN selection wedges of Lacy et al. (2007) and Stern et al. (2005) along with the IRAC power-law locus (the line on which a source with a perfect IRAC power-law SED would fall), where circles denote powerlaw slopes of $\alpha=-0.5$ to $-3.0\left(f_{v} \propto v^{\alpha}\right)$. While the power-law locus itself extends to bluer slopes, luminous AGNs are expected to display red slopes of $\alpha \leqslant-0.5$ (Alonso-Herrero et al. 2006; Donley et al. 2007).

In both Lacy et al. (2004, 2007) and Stern et al. (2005) color space, purely star-forming templates generally avoid the 


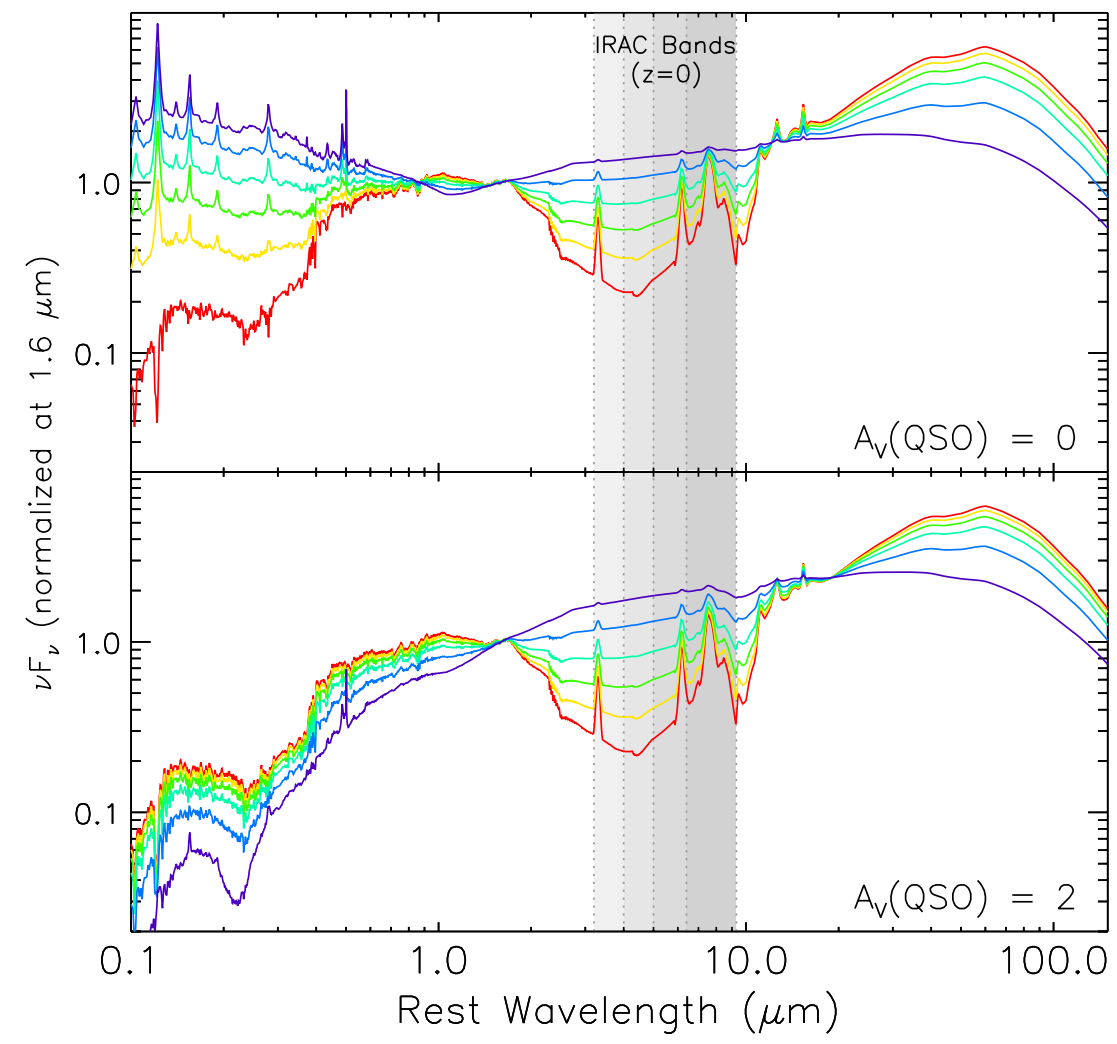

Figure 1. Composite SEDs constructed using the QSO1 and M82 templates of Polletta et al. (2008), scaled to give 1-10 $\mu$ m AGN contributions of 0\% (red in the online journal) to $95 \%$ (purple in the online journal). The final SEDs have been normalized at $1.6 \mu \mathrm{m}$. In the lower panel, we apply an extinction of $A_{\mathrm{V}}=2$ to the QSO1 SED using the Draine (2003) extinction law. The four IRAC bands at $z=0$ are shaded. While luminous unobscured and obscured AGNs have very different UV-optical SEDs, luminous AGNs should display a red MIR power-law SED regardless of obscuration.

(A color version of this figure is available in the online journal.)

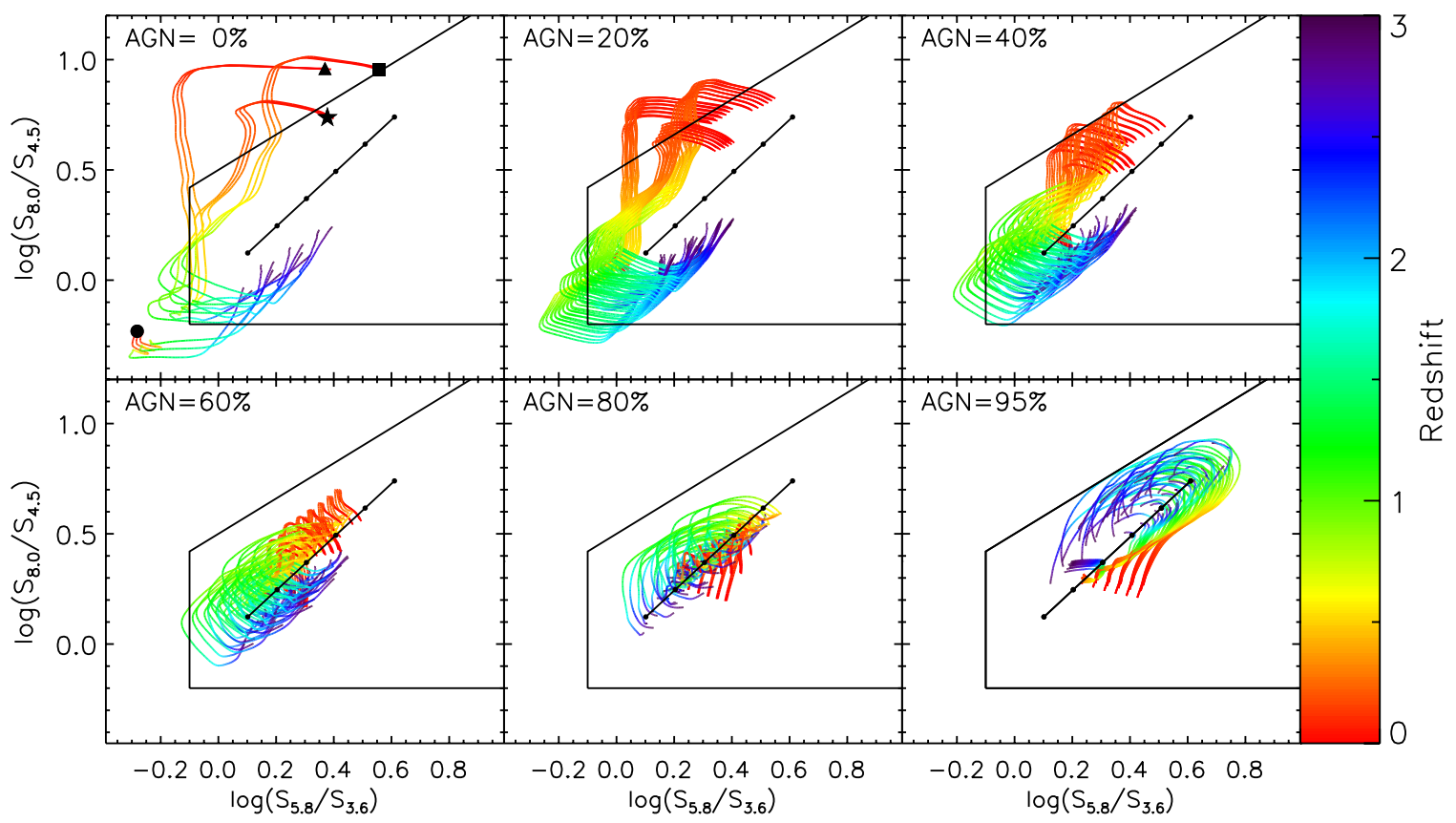

Figure 2. Predicted $z=0-3$ IRAC colors of AGN/galaxy composite SEDs in Lacy et al. (2004, 2007) color space, where the AGN fraction is defined between 1 and $10 \mu \mathrm{m}$. The star-forming templates represent the ULIRG IRAS 22491 (square; Polletta et al. 2008), the starburst M82 (star; Polletta et al. 2008), a normal star-forming spiral galaxy (triangle; Dale \& Helou 2002), and an elliptical galaxy (circle; Polletta et al. 2008), where large symbols mark each family of purely star-forming templates at $z=0$. The AGN template is the QSO1 template of Polletta et al. (2008). Additional extinctions of $A_{\mathrm{V}}=0-2$ and $A_{\mathrm{V}}=0-20$ are applied to the star-forming and AGN components, respectively. The wedge is the AGN selection region of Lacy et al. (2007), and the line represents the power-law locus from $\alpha=-0.5$ (lower left) to $\alpha=-3.0$ (upper right). While the templates of purely star-forming galaxies avoid the power-law locus, they enter the current AGN selection region at both low and high redshifts. As the AGN's contribution to the MIR emission increases, the SEDs move inward and redward toward the power-law locus.

(A color version of this figure is available in the online journal.) 


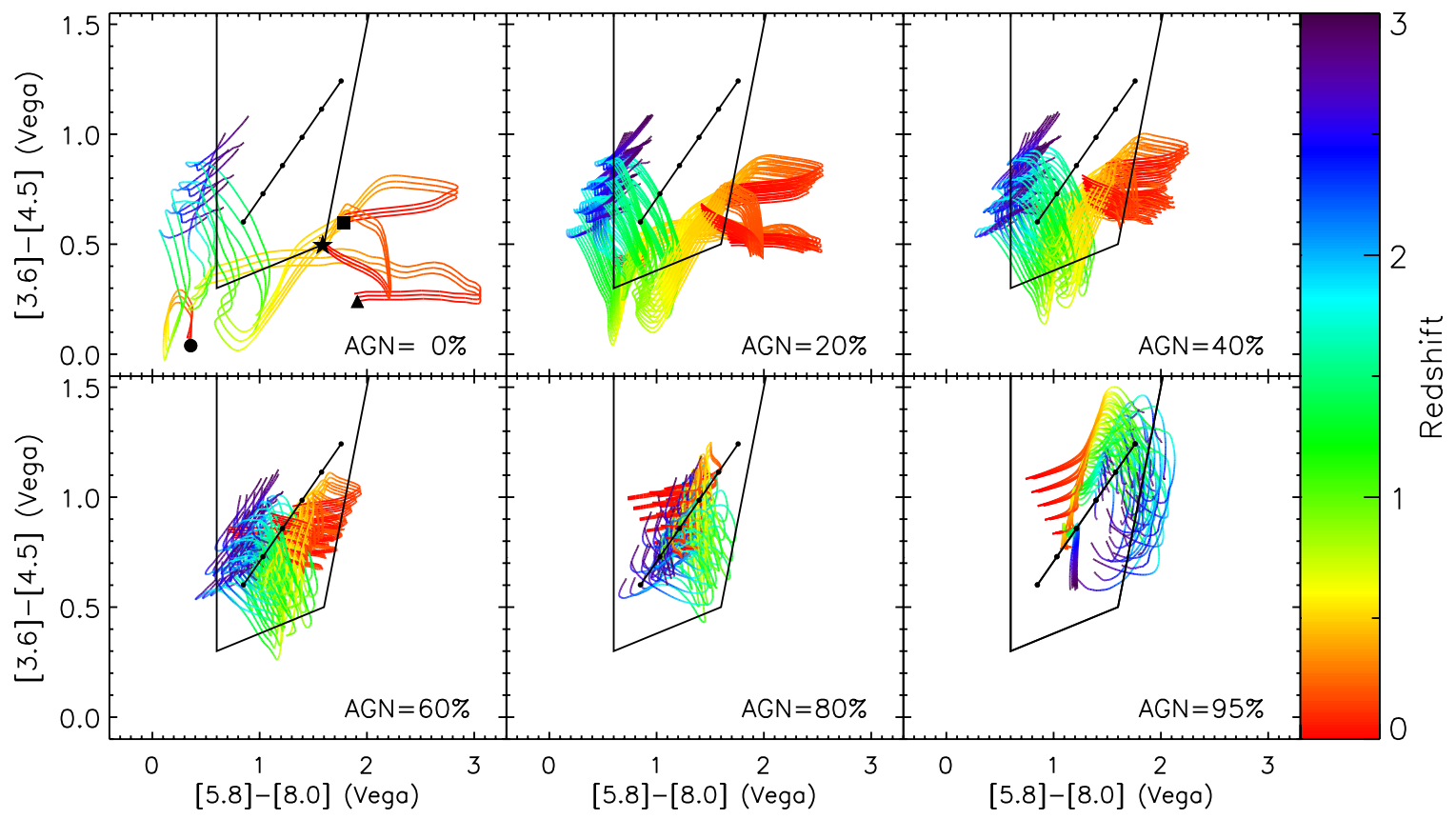

Figure 3. Same as Figure 2, but for Stern et al. (2005) color space. The wedge is the AGN selection region of Stern et al. (2005).

(A color version of this figure is available in the online journal.)

region surrounding the power-law locus, at least out to $z \sim 3$ (the potential for contamination by higher redshift galaxies will be discussed in Section 8). As the AGN becomes increasingly dominant, the IRAC colors then shift inward and redward toward the power-law locus. Generally speaking, the more luminous the AGN, the redder the IRAC colors. A source's precise location in IRAC color space, however, will depend not only on the relative AGN/host contributions, but on its redshift, the reddening of its host and AGN components, and the host galaxy type.

\subsection{IRAC Colors of Purely Star-forming Galaxies}

While the purely star-forming templates shown in Figures 2 and 3 lie outside of the power-law region in IRAC color space, they enter the Lacy et al. (2004, 2007) and Stern et al. (2005) AGN selection wedges at both low and high redshift (Barmby et al. 2006; Donley et al. 2007, 2008). Thanks to recent Spitzer/ IRS spectroscopic campaigns in fields with deep IRAC data, however, we need no longer rely solely on templates to constrain the IRAC colors of purely star-forming galaxies.

The IRAC colors of Spitzer/IRS sources classified as pure starbursts are plotted in Figure 4 (see Table 1 for details). While each IRS study uses slightly different criteria for identifying purely star-forming galaxies, we standardize this definition by requiring that $\mathrm{EW}(6.2 \mu \mathrm{m}) \geqslant 0.4$ when the $6.2 \mu \mathrm{m}$ polycyclic aromatic hydrocarbon (PAH) feature lies within the observed bandpass (Weedman \& Houck 2009) and EW(11.3 $\mu \mathrm{m})>0.8$ when it does not (Dasyra et al. 2009), where EW is the equivalent width. We exclude any source flagged as having blended IRAC photometry, and plot for comparison the colors of sources that have not been observed with IRS, but that meet the $1.5<z<3$ star-forming IRAC criteria of Huang et al. (2009).

The IRAC colors of the IRS star-forming galaxies are generally consistent with the predictions from Figures 2 and 3. In Lacy et al. (2004, 2007) color space, low-redshift galaxies lie predominantly above the AGN wedge, though some fall just within the AGN selection region. At $0.5<z<1.5$, they fall both to the left of and within the AGN wedge, and at $z>1.5$, the vast majority of star-forming galaxies lie within the AGN selection region. In Stern et al. (2005) color space, most low- and moderate-redshift star-forming galaxies fall below the selection region, and many of the high-redshift galaxies lie to the left of the AGN wedge. However, a significant fraction of the Huang et al. (2009) $1.5<z<3$ star-forming candidates scatter into the AGN selection region, as do several moderateand high-redshift IRS-selected starbursts.

With the exception of a $z=0.73$ ULIRG from Dasyra et al. (2009; shown by a cross near the $\alpha \sim-1.0$ power-law locus), the star-forming galaxies lie well away from the power-law locus, especially at $\alpha \leqslant-1.0$, and in Lacy et al. (2004) color space. The IRS spectrum of this source displays strong PAH emission with $\operatorname{EW}(11.3 \mu \mathrm{m})=1.43 \pm 0.07$ (Dasyra et al. 2009), and a low $\mathrm{S} / \mathrm{N}$ optical spectrum shows strong [O $\mathrm{II}], \mathrm{H} \beta$, and [O III] emission but no obvious high-ionization lines (Papovich et al. 2006). At $z=0.73$, however, neither the optical nor the MIR spectral coverage extend into the $1-5 \mu \mathrm{m}$ rest-frame regime sensitive to AGN-heated dust. Given the available data, it is therefore unclear whether this source's power-law-like IRAC SED is due to a contribution from AGN-heated dust, or whether it is a true star-forming interloper. Nevertheless, it is clear from Figures 2, 3, and 4 that while the current AGN selection regions inadequately separate AGNs from moderate- to highredshift star-forming galaxies, luminous AGNs should occupy a well-defined region of color space with minimal star-forming contamination. To test these predictions and better constrain the region of IRAC color space that can reliably be used for AGN selection, we turn to COSMOS.

\section{COSMOS DATA AND SAMPLE SELECTION}

The $2 \mathrm{deg}^{2}$ COSMOS field is characterized by both deep and wide coverage across much of the electromagnetic spectrum. We focus here on the XMM-Newton and Spitzer/IRAC data sets that cover the full survey area (Hasinger et al. 2007; Sanders et al. 2007), as well as the deeper Chandra data available over the central $0.9 \mathrm{deg}^{2}$ (Elvis et al. 2009). We also make use of the 

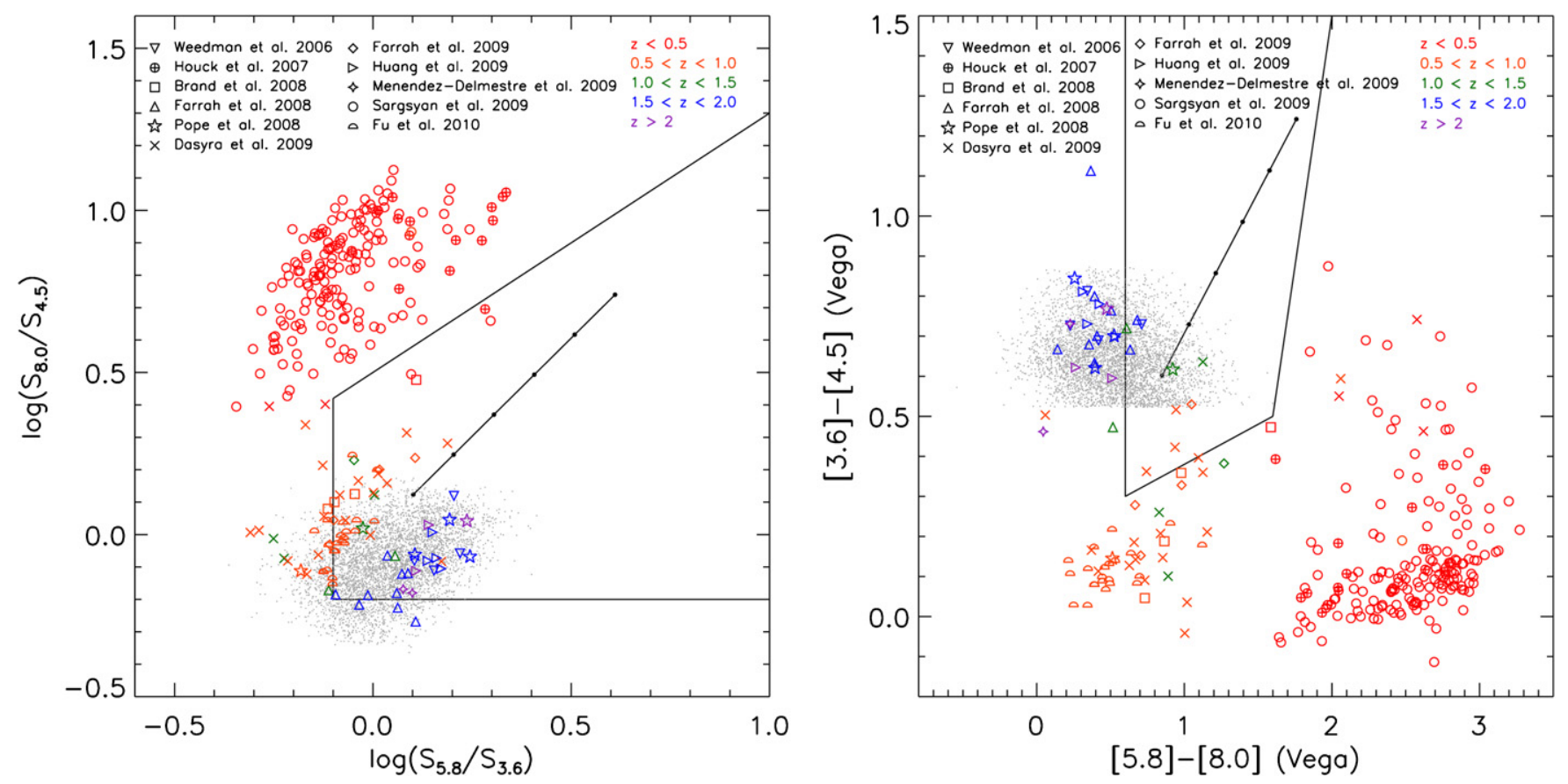

Figure 4. IRAC colors of pure starbursts, as determined via Spitzer/IRS spectroscopy (large symbols). The small gray points represent sources that have not been observed with IRS, but that meet the $1.5<z<3$ star-forming IRAC criteria of Huang et al. (2009). The wedges show the current AGN selection regions of Lacy et al. (2007, left) and Stern et al. (2005, right), and the line shows the power-law locus from $\alpha=-0.5$ to $\alpha=-3.0$. A significant fraction of purely star-forming galaxies lie within the current AGN selection regions, particularly at high redshift.

(A color version of this figure is available in the online journal.)

Table 1

Pure Starbursts with IRS Spectra

\begin{tabular}{lcccc}
\hline \hline Number & Mean $z$ & Sample & Fields & Selection \\
\hline 16 & $0.06 \pm 0.04$ & Houck et al. (2007) & NDWFS & $f_{24}>10 \mathrm{mJy}$ \\
182 & $0.13 \pm 0.10$ & Sargsyan \& Weedman (2009) & SWIRE, NDWFS & $z<0.5$ starbursts \\
22 & $0.71 \pm 0.06$ & Fu et al. (2010) & COSMOS & $f_{24}>0.7 \mathrm{mJy}, z \sim 0.7$ \\
24 & $0.74 \pm 0.22$ & Dasyra et al. (2009) & SFLS & $f_{24}>0.9 \mathrm{mJy}$ \\
4 & $0.75 \pm 0.29$ & Brand et al. (2008) & NDWFS & $70 \mu$ m selected, optically faint \\
5 & $0.86 \pm 0.14$ & Farrah et al. (2009) & SWIRE & $70 \mu$ m selected, optically faint \\
11 & $1.63 \pm 0.12$ & Farrah et al. (2008) & SWIRE & IRAC bump selected \\
6 & $1.75 \pm 0.60$ & Pope et al. (2008) & SMGs \\
4 & $1.82 \pm 0.10$ & Weedman et al. (2006) & SWIRE & IRAC bump selected \\
6 & $2.05 \pm 0.48$ & Huang et al. (2009) & EGS & IRAC bump selected \\
2 & $2.59 \pm 0.63$ & Menéndez-Delmestre et al. (2009) & SWIRE & SMGs \\
\hline
\end{tabular}

intermediate- and broadband optical-NIR data used to construct photometric redshifts for the X-ray detected and normal galaxy populations (Salvato et al. 2009, 2011; Ilbert et al. 2009; Capak et al. 2007; P. Capak et al., in preparation).

\subsection{IRAC}

The S-COSMOS IRAC data cover the full $2.3 \mathrm{deg}^{2}$ COSMOS field to $1200 \mathrm{~s}$ depth, reaching $5 \sigma$ sensitivities of $0.9,1.7,11.3$, and $14.6 \mu \mathrm{Jy}$ in the $3.6,4.5,5.8$, and $8.0 \mu \mathrm{m}$ bands, respectively (Sanders et al. 2007). The IRAC sample used here contains the 26,251 non-flagged IRAC sources that lie in the $1.73 \mathrm{deg}^{2}$ region of COSMOS with high-quality unmasked optical-NIR data (Ilbert et al. 2009, 2010), have an optical/NIR counterpart within a $2^{\prime \prime}$ search radius, are not flagged as stars by Ilbert et al. (2009) or McCracken et al. (2010), have a photometric or spectroscopic redshift, and meet the $5 \sigma$ sensitivity limits of Sanders et al. (2007) in each of the four IRAC bands. The aperture-corrected IRAC photometry was calculated in 1".9 apertures using SExtractor (Sanders et al. 2007).

\section{2. $X M M$}

Our primary sample of X-ray-selected AGNs is drawn from the $\sim 40$ ks XMM-COSMOS survey (Hasinger et al. 2007; Cappelluti et al. 2009; Brusa et al. 2010). While the Chandra data of Elvis et al. (2009) reach greater depths over the central $0.9 \mathrm{deg}^{2}$ and will be used later in the paper, the larger area of the XMM survey better samples the population of luminous yet low space density AGNs, resulting in roughly equal numbers of Seyfert galaxies $\left(\log L_{\mathrm{x}}\left(\mathrm{erg} \mathrm{s}^{-1}\right) \leqslant 44\right)$ and QSOs $\left(\log L_{\mathrm{x}}\left(\mathrm{erg} \mathrm{s}^{-1}\right) \geqslant 44\right)$.

The full XMM-COSMOS sample of Brusa et al. (2010) contains 1797 sources, $93 \%$ of which have a spectroscopic or photometric redshift indicative of an extragalactic source. (Of the remaining sources, $5.5 \%$ are Galactic stars, and $1.5 \%$ lack a redshift estimate.) We restrict our $X M M$ sample to the sources that lie within the $1.73 \mathrm{deg}^{2}$ region of COSMOS with deep, uniform, and unmasked optical-NIR data (Ilbert et al. 2009), have a reliable optical/NIR counterpart in Brusa et al. (2010), 
are not flagged as stars in Brusa et al. (2010), Ilbert et al. (2009), or McCracken et al. (2010), have a photometric or spectroscopic redshift, and have high-quality IRAC counterparts that meet or exceed the COSMOS $5 \sigma$ sensitivity in each of the four IRAC bands (Sanders et al. 2007). Applying all but the IRAC detection criterion gives a sample of 1183 sources, $12 \%$ of which are excluded in our final sample of 1039 XMM sources with highsignificance IRAC counterparts.

Of the $X M M$ sources, $63 \%$ have a spectroscopic redshift as compiled by Brusa et al. (2010) and the remaining $37 \%$ have an AGN-specific photometric redshift from Salvato et al. (2009). To constrain the IRAC properties of the XMM sample, we use the IRAC flux densities from Ilbert et al. (2009; as given by Brusa et al. 2010), which have been re-extracted at the positions of the $X M M$ sources to minimize the effects of blending. To permit comparison with the $3^{\prime \prime}$ optical-NIR aperture photometry, Ilbert et al. (2009) multiplied all of the IRAC flux densities by 0.75 . Here, we undo this correction to recover the total IRAC flux densities.

\subsection{Chandra}

The Chandra-COSMOS coverage reaches $160 \mathrm{ks}$ depth over $0.5 \mathrm{deg}^{2}$ and $80 \mathrm{ks}$ depth over an additional $0.4 \mathrm{deg}^{2}$ (Elvis et al. 2009). The full catalog of Chandra sources contains 1761 sources, 1010 of which have optical/NIR and IRAC counterparts from Elvis et al. (2009) and F. Civano et al. (in preparation) that meet the criteria described above. Of these, $56 \%$ are also $X M M$ sources, and $61 \%$ have spectroscopic redshifts. For the remaining Chandra sources, we adopt the AGN-specific photometric redshifts of Salvato et al. (2011).

\subsection{Optical-NIR Photometry and Redshifts}

Optical and NIR photometry are taken from the intermediateand broadband catalogs of Capak et al. (2007), Ilbert et al. (2009), McCracken et al. (2010), and P. Capak et al. (in preparation). We apply the band-specific systematic offsets given by Ilbert et al. (2009), which correct for errors in the zero points and minimize the systematic scatter in the photometric redshifts, and we aperture correct the photometry using the band-independent auto-offset aperture corrections.

High-confidence spectroscopic redshifts and spectral classifications were compiled from the Sloan Digital Sky Survey (SDSS; Kauffmann et al. 2003; Adelman-McCarthy et al. 2006), the Magellan/IMACS and MMT observing campaigns of Prescott et al. (2006), Trump et al. (2007), and Trump et al. (2009), zCOSMOS (Lilly et al. 2007), and subsequent Keck runs. Photometric redshifts for non-X-ray sources are taken from Ilbert et al. (2009), whereas AGN-specific photometric redshifts for the $X M M$ and Chandra samples were drawn from Salvato et al. (2009, 2011), respectively.

\section{POWER-LAW SELECTION: A STARTING POINT}

IRAC power-law selection identifies sources that lie near the power-law locus in color space, and therefore selects only the most secure AGN-dominated candidates from the IRAC AGN wedges (e.g., Alonso-Herrero et al. 2006; Donley et al. 2007, 2008; Park et al. 2010). For a source to be selected as a power-law AGN, a line of slope $\alpha \leqslant-0.5$ must provide a good fit to its logarithmic IRAC photometry $\left(f_{v} \propto v^{\alpha}\right.$, or $\left.\log f_{v}=\alpha \log v+b\right)$. The goodness of fit is measured by the $\chi^{2}$ probability, $P_{\chi}$, with a limit of $P_{\chi} \geqslant 0.1$ (Donley et al. 2007, 2008).

IRAC power-law selection therefore depends not only on the IRAC flux densities used for color-color selection, but also on the IRAC errors typically derived using SExtractor. Unfortunately, SExtractor tends to underestimate photometric uncertainties, as it does not account for the correlated sky noise present in mosaicked data (e.g., Gawiser et al. 2006; Barmby et al. 2008). Furthermore, the cataloged measurement errors do not account for the IRAC calibration uncertainty, which is at best $3 \%$ and is at worst $10 \%$ when the sub-pixel response and the array-location-dependent changes in pixel solid angle and spectral response are not taken into account (Reach et al. 2005). The SExtractor errors can therefore be as low as $\Delta f_{v} / f_{v}=$ $0.1 \%$ for our IRAC sample, with the brightest IRAC sources exhibiting the most underestimated errors.

Using such highly underestimated errors, it becomes nearly impossible to obtain a statistically acceptable fit to a given SED, even when the SED appears to closely match the photometry. As an example, consider the $25 X M M$ sources that lie in the immediate vicinity (within $0.025 \mathrm{dex}$ ) of the $\alpha=-1.0$ powerlaw locus. Using the flux densities and errors from the official COSMOS IRAC catalog, the power-law criterion is met by only $30 \%$ of the fainter half of the sample and by none of the brighter half of the sample.

This effect is further illustrated in Figure 5, where we plot the IRAC colors of the XMM- and S-COSMOS samples and identify the sources selected as power-law galaxies using the cataloged errors and those selected as power-law galaxies when additional uncertainties of $3 \%, 5 \%, 10 \%$, and $15 \%$ are added to the cataloged errors. To quantify the dependency of power-law selection on the IRAC errors, we define a box around the powerlaw locus in Lacy et al. $(2004,2007)$ color space that encloses nearly all of the XMM sources with red $(\alpha \leqslant-0.5)$ IRAC colors (see Figure 5). If we were to select IRAC power-law galaxies using the cataloged errors, we would recover only $5 \%$ of the XMM sources in the power-law box. Adding additional uncertainties of $3 \%, 5 \%, 10 \%$, and $15 \%$ to the IRAC errors increases this fraction to $41 \%, 69 \%, 96 \%$, and $98 \%$, respectively.

Because the sources in the full IRAC sample tend to be fainter than those in the $X M M$ sample, they display more scatter in IRAC color space. Nonetheless, we observe a similar trend in power-law selection: $12 \%, 40 \%, 62 \%, 91 \%$, and $96 \%$ of the sources in the power-law box are selected as power-law galaxies using the cataloged errors and additional uncertainties of $3 \%$, $5 \%, 10 \%$, and $15 \%$, respectively.

Adjustments to the SExtractor-derived IRAC errors are clearly required to identify complete samples of AGNs that lie near the power-law locus in color space. In previous work, we have assumed an overall IRAC calibration uncertainty of $10 \%$ to recover the majority of X-ray sources with red IRAC colors (Alonso-Herrero et al. 2006; Donley et al. 2007, 2008). While this likely overestimates the true errors on the flux, this assumption facilitates the selection of sources with small deviations from a perfect power-law SED. As shown in Figure 5, however, these red, power-law-dominated AGNs occupy a well-defined region of Lacy et al. (2004, 2007) IRAC color space. Power-law galaxies could therefore be selected on the basis of their IRAC colors alone, thus eliminating the dependence of power-law selection on poorly constrained photometric errors and assumed uncertainties.

In contrast, power-law galaxies cannot be cleanly identified using color-color cuts in Stern et al. (2005) color space. 

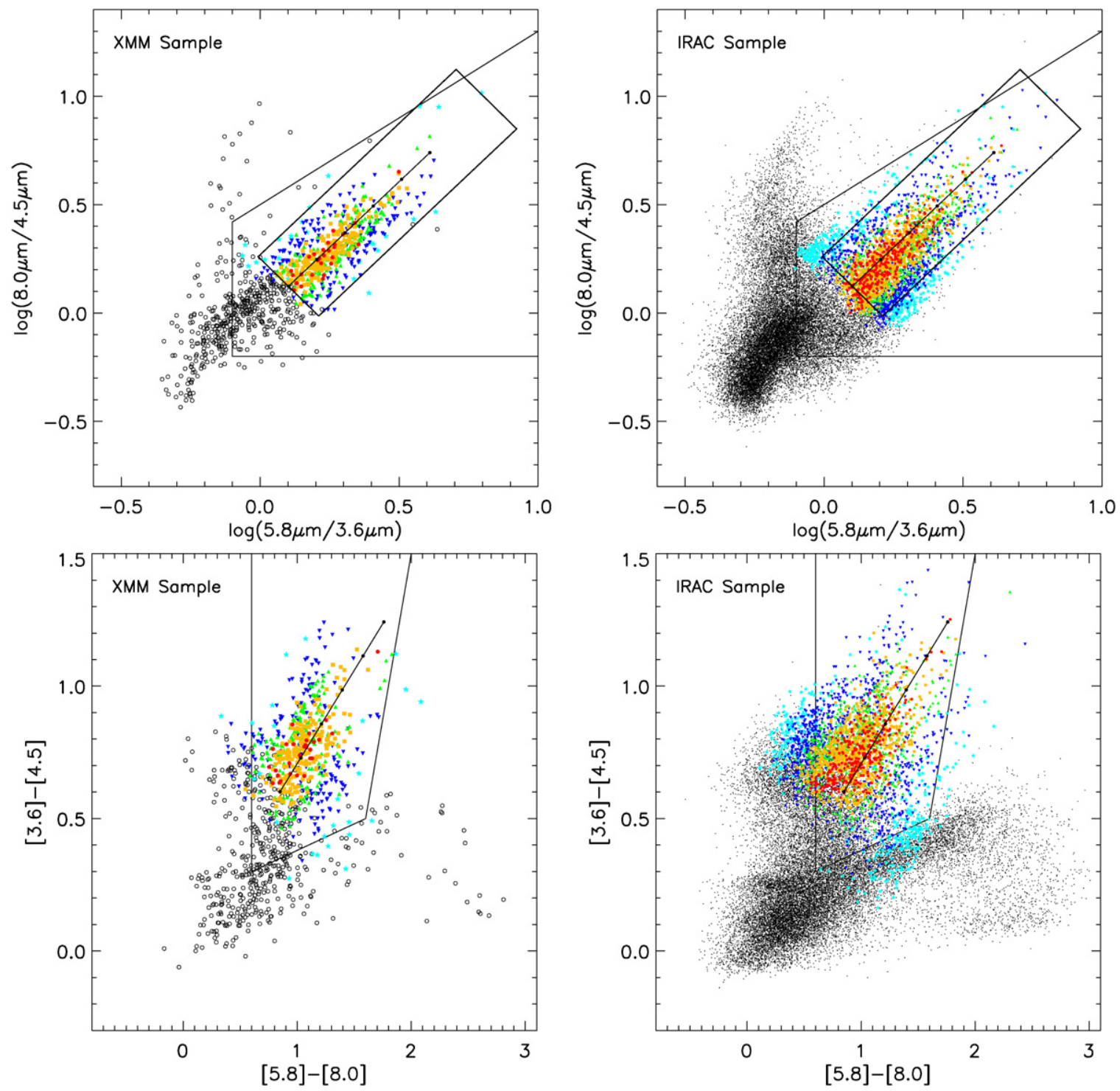

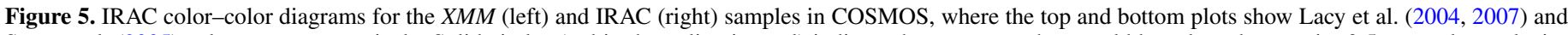

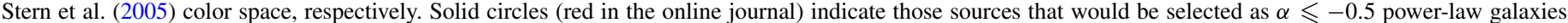

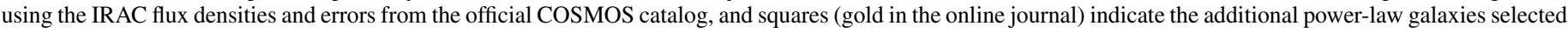

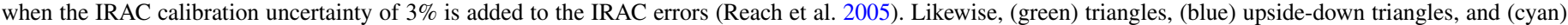

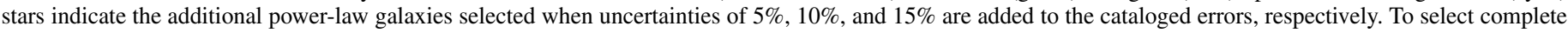

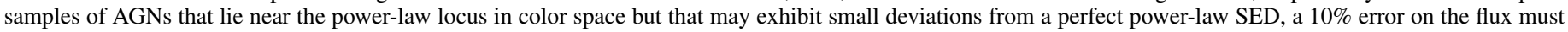
be assumed.

(A color version of this figure is available in the online journal.)

This increased scatter arises from the use of adjacent bands (3.6/4.5 $\mu \mathrm{m}$ and 5.8/8.0 $\mu \mathrm{m})$, which minimizes the wavelength baseline, increases the sensitivity to small variations in the SEDs, and groups together the two low-sensitivity IRAC channels (5.8 and $8.0 \mu \mathrm{m}$; see also Donley et al. 2007, 2008; Richards et al. 2009). We therefore focus below on the Lacy et al. (2004, 2007) representation of IRAC color space, although we will continue to show relevant plots in both representations to motivate the need for new selection criteria.

We take as a starting point for these revised IRAC color-color cuts the $\alpha \leqslant-0.5$ power-law box defined above. Not only does this region enclose nearly all $X M M$ sources with red IRAC colors, but it also tightly encloses the AGN-dominated templates shown in Figure 2. While this power-law box should therefore be highly complete, at least to luminous AGNs that dominate the light from their hosts, high-redshift star-forming galaxies may enter the bluest portions of the power-law box (see Figure 4). In the sections that follow, we therefore use the COSMOS XMM, Chandra, and IRAC samples, as well as samples of high-redshift galaxies, to further refine this revised AGN selection region and to quantify its completeness and reliability.

\section{XMM SAMPLE: PROPERTIES}

To constrain the efficiency and completeness of IRAC selection and test for any trends in AGN properties across IRAC color space, we first determine the intrinsic obscuration and $\mathrm{X}$-ray luminosity of the $X M M$-COSMOS sample.

\subsection{AGN Type: Optical and X-Ray Classification}

AGNs can be divided into type 1 (unobscured) or type 2 (obscured) classes using a variety of criteria, including optical 
spectral classification, optical SED fitting, IR-to-optical flux ratios, X-ray spectral fitting, and X-ray hardness or flux ratios (for a discussion of the agreement between classification schemes, see Trouille et al. 2009). Here, we focus on two methods. For the first, we adopt the approach of Brusa et al. (2010) and use the optical spectral classification when available and the best-fit broadband SED type from Salvato et al. (2009) otherwise, and refer to the resulting classes as "optical type 1" and "optical type 2." Of the XMM sources with type 1 and type 2 spectra, $85 \%-90 \%$ are also best fit by type 1 and type 2 SEDs, respectively, indicating that these two variations on optical classification are well matched. Using this approach, we find an optical type 2 fraction of $58 \%$ for the $X M M$ sample.

For the second approach, we turn to the X-ray and use each source's redshift, hard to soft X-ray flux ratio, and an assumed intrinsic X-ray photon index of $\Gamma=1.8$ (Tozzi et al. 2006) to estimate its intrinsic column density, $N_{\mathrm{H}}$. We then group sources into "X-ray type 1" and "X-ray type 2" classes using a cut of $N_{\mathrm{H}}=10^{22} \mathrm{~cm}^{-2}$. For the brightest sources in our sample, we compare our estimated column with that derived from X-ray spectral fitting (Mainieri et al. 2007). For the 24 sources with measurable columns from both techniques, we find a typical absolute scatter of $\log N_{\mathrm{H}}\left(\mathrm{cm}^{-2}\right)=0.28$ and a mean offset of $\Delta \log N_{\mathrm{H}}\left(\mathrm{cm}^{-2}\right)=0.02$. The agreement between our simple flux ratio method and the full spectral fitting is therefore generally good.

Using the flux-ratio-derived column density, we measure X-ray types for $\sim 3 / 4$ of our sample and find an X-ray type 2 fraction of $52 \%$. Of the remaining sources, $90 \%$ are detected in only the soft band but have upper limits on $N_{\mathrm{H}}$ that are potentially consistent with the type 2 class (due to the comparably low sensitivity of the hard X-ray band), and $10 \%$ are detected in only the hard band but have lower limits on $N_{\mathrm{H}}$ that are potentially consistent with the type 1 class. This leads to ambiguous classifications.

Comparing the X-ray and optical types, we find that $65 \%$ of the optically classified AGNs have matching X-ray types when the latter is available. While this fraction is far from $100 \%, \sim$ $20 \%-30 \%$ of AGNs have mismatched optical and X-ray types even when classifications are made using deep X-ray data and optical spectra, an apparent discrepancy whose proposed explanations include spectral variability, host galaxy dilution, and disappearance of the broad-line region in AGNs accreting at $L / L_{\text {Edd }} \leqslant 0.01$ (e.g., Tozzi et al. 2006; Page et al. 2006; Caccianiga et al. 2007; Trouille et al. 2009; Trump et al. 2009, 2011a, 2011b). To further complicate matters, observational scatter in the measured X-ray fluxes, when combined with the strict dividing line in column density between X-ray type 1 and type 2 sources, leads to discrepant X-ray types for $15 \%-30 \%$ of the sources in our sample detected by both Chandra and XMM.

\subsection{Intrinsic X-Ray Luminosity}

For the $70 \%$ of $X M M$ sources in our sample with a hard-band detection, we estimate the intrinsic (e.g., absorption-corrected) rest-frame $2-10 \mathrm{keV}$ luminosities using the column densities estimated above and the observed $2-10 \mathrm{keV}$ fluxes. While we can place only lower limits on the columns of the $13 \%$ of sources that lack soft-band detections (with a median value of $\left.\log N_{\mathrm{H}}\left(\mathrm{cm}^{-2}\right) \geqslant 22.7\right)$, their estimated intrinsic luminosities would increase by an average of only $\sim 0.4 \mathrm{dex}$ if all such sources were Compton-thick with $\log N_{\mathrm{H}}\left(\mathrm{cm}^{-2}\right)=24$ (and by only $\sim 0.1$ dex if $\left.\log N_{\mathrm{H}}\left(\mathrm{cm}^{-2}\right)=23.5\right)$. In contrast, because of the comparably low sensitivity of the hard X-ray band, the majority (77\%) of sources detected only in the soft band have column density upper limits in excess of $\log N_{\mathrm{H}}\left(\mathrm{cm}^{-2}\right)=22$, and $24 \%$ have upper limits greater than $\log N_{\mathrm{H}}\left(\mathrm{cm}^{-2}\right)=23$ (assuming an intrinsic X-ray spectrum with $\Gamma=1.8$ ). Such high columns can translate into order-of-magnitude corrections to the intrinsic luminosity estimated from the observed soft-band flux, so we do not attempt to estimate intrinsic luminosities for the $30 \%$ of $X M M$ sources detected only in the soft band.

All of the $X M M$ sources with a hard-band detection have both optical and X-ray classifications, with optical and X-ray type 2 fractions of $57 \%$ and $58 \%$, respectively, and $68 \%$ have spectroscopic redshifts. For sources with a measurable column or those with only a hard-band detection, we estimate the absorption-corrected rest-frame luminosity by again assuming an intrinsic $\Gamma$ of 1.8 . For sources with no measurable column (i.e., those that are softer than our assumed $\Gamma$ of 1.8), we use the Galactic absorption-corrected hard-to-soft flux ratio to calculate the observed photon index, $\Gamma$, which we then use to determine the rest-frame luminosity.

\subsection{Broadband SEDs}

Before discussing the IRAC colors of the XMM sample, we examine the median SEDs of sources binned by X-ray or optical type and X-ray luminosity. Doing so sheds light on the nature of AGNs of different classes, and also illustrates the inherent differences and biases in the classification schemes which will affect our later interpretation.

We plot in Figure 6 the best-fit templates to the median SEDs of the X-ray and optical type 1 and type 2 populations, as a function of intrinsic X-ray luminosity. To create these templates, we shift each individual SED into the rest frame and normalize it to $1 \mu \mathrm{m}$ (the approximate location of both the inflection point seen in luminous AGNs and the midpoint of the stellar bump prominent in lower-luminosity AGNs). We then calculate the median SED in bins of $\log \Delta \lambda(\AA)=0.1$ for all bins containing at least 10 points. This procedure gives a low-resolution median SED, albeit one with occasional gaps in wavelength coverage. To provide a clearer representation of the median SEDs, we then fit each median SED with all possible combinations of one of six AGN templates (Sey1.8, Sey2, QSO1, BQSO1, TQSO1, QSO2) and one of 14 star-forming templates (comprising all elliptical and spiral templates as well as the starburst/ULIRG templates of N6090, M82, Arp220, and I22491) from Polletta et al. (2008), choosing as the final fit the template combination with the lowest reduced $\chi^{2}$. To account for obscuration, we allow for independent reddening of the AGN and star-forming templates using the Draine (2003) extinction law.

The SEDs shown in Figure 6 should be interpreted as rough guides to the overall behavior of the $X M M$ sources. In general, they are far more highly sampled in the UV/optical than in the MIR, and they do not reflect the intrinsic variation seen in any given class of AGNs (for more detailed SED analyses, see Salvato et al. 2009; Hao et al. 2010; Lusso et al. 2010; Trump et al. 2011a; Lusso et al. 2011; M. Elvis et al., in preparation). That said, they clearly illustrate the redder MIR colors of more luminous AGNs (see also Figures 1, 2, and 3) and confirm that this trend is relatively insensitive both to obscuration and to the classification scheme (X-ray versus optical).

\subsubsection{Differences between X-Ray and Optical Classification Schemes}

While the MIR SEDs plotted in Figure 6 are relatively insensitive to the obscuration scheme, X-ray and optical AGN classification returns samples with different UV-optical SEDs. 

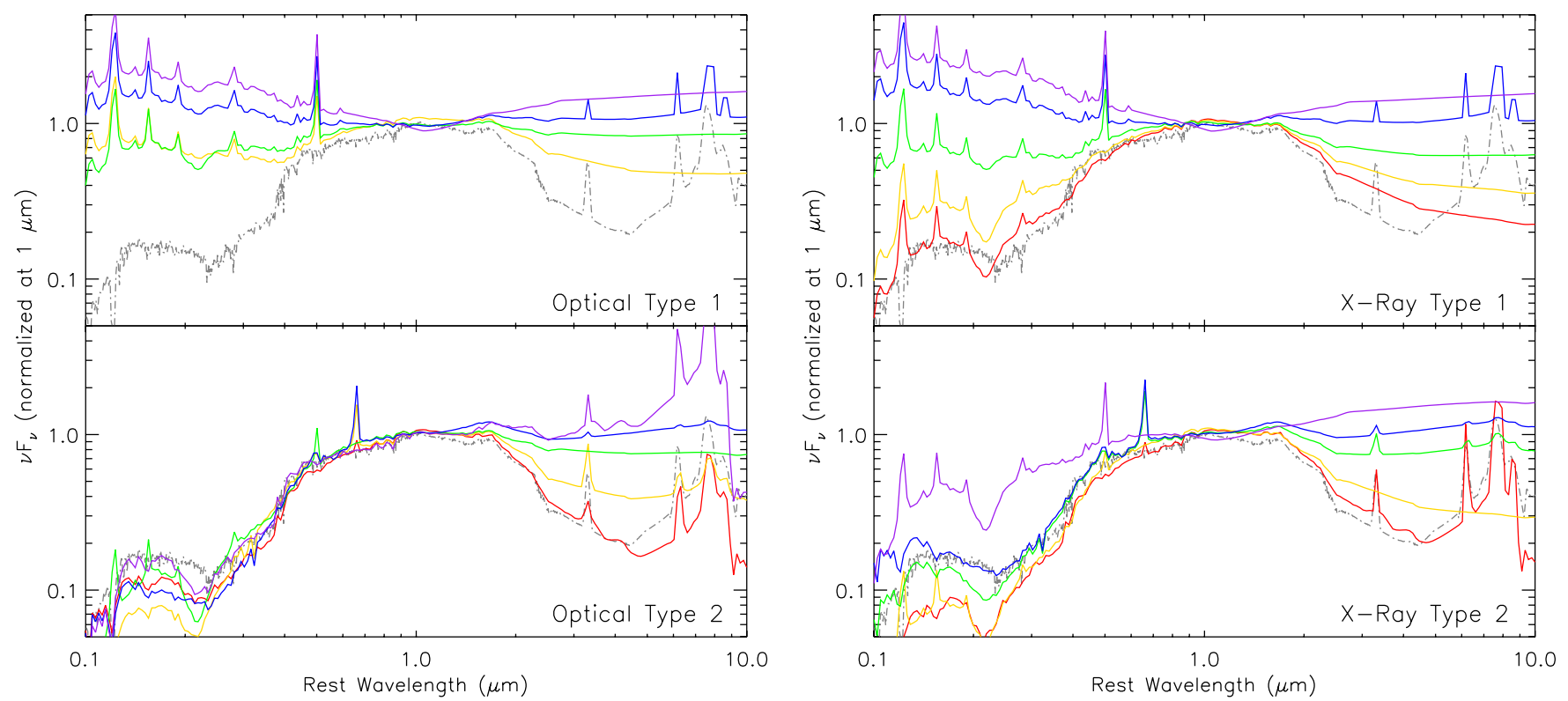

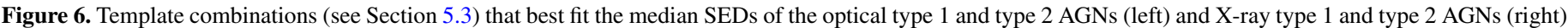

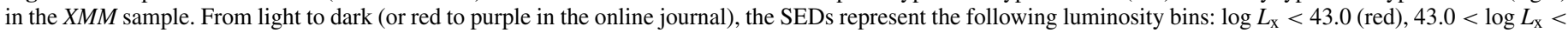

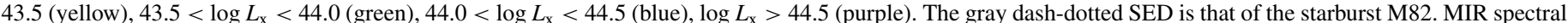

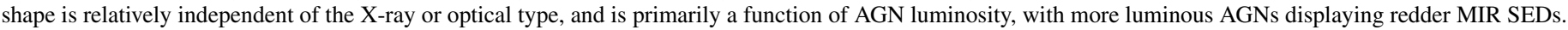
The differences in the SEDs of X-ray and optically classified AGNs can be explained by the selection effects and biases discussed in Section 5.3.1.

(A color version of this figure is available in the online journal.)

This apparent discrepancy can be attributed in part to the physically distinct samples identified by the two criteria, and in part to selection biases. For a source to be classified as "optical type 1," it must display either broad optical emission lines or have an SED best fit by an unobscured quasar template relatively flat in $v F_{v}$. All remaining sources, including those with narrow optical emission lines, those with no emission lines yet X-ray luminosities exceeding $2 \times 10^{42} \mathrm{erg} \mathrm{s}^{-1}$ (e.g., optically dull AGNs; Moran et al. 2002; Comastri et al. 2002), and those lacking optical spectra yet best fit by a galaxy-dominated template are classified as "optical type 2." In the absence of highquality spectra, low-luminosity type 1 AGNs (e.g., Seyfert 1 galaxies) with host-dominated SEDs are therefore likely to be misclassified as optical type 2 AGNs (see also the discussion in Brusa et al. 2010).

However, the vast majority (88\%) of low-luminosity (log $\left.L_{2-10 \mathrm{keV}}\left(\mathrm{erg} \mathrm{s}^{-1}\right)<43.5\right) \mathrm{X}$-ray type 1 AGNs in our XMM sample have robust optical spectra, yet $77 \%$ lack broad optical emission lines. While we cannot rule out the possibility that the host galaxies of these low-luminosity AGNs have diluted their broad emission lines (e.g., Page et al. 2006; Caccianiga et al. 2007; Civano et al. 2007), Trump et al. (2011a) show that the X-ray unobscured narrow-line AGNs in $X M M$-COSMOS have low accretion rates of $L / L_{\mathrm{Edd}}<10^{-2}$, and may therefore be fueled by radiatively inefficient accretion flows incapable of supporting a broad-line region. The X-ray type 1, yet optical type 2 , classification may therefore correctly represent the intrinsic nature of these low-luminosity AGNs, whose seemingly discrepant properties give rise to many of the observed differences in Figure 6.

$\mathrm{X}$-ray classification is less sensitive to accretion rate and host galaxy properties, but is subject to a redshift-dependent bias. At low redshift, the soft X-ray band $(0.5-2 \mathrm{keV})$ is sensitive to relatively low obscuring columns. At high redshift, however, low to moderate column densities become poorly constrained as the observed $\mathrm{X}$-ray bands sample the higher energy restframe emission less subject to intrinsic obscuration. Small observational uncertainties in X-ray flux therefore translate to large uncertainties in column density, and because low columns are far more poorly constrained than high columns, type 1 AGNs are far more likely to be misclassified as type 2 AGNs than vice versa. We illustrate and quantify this well-known effect in more detail in the Appendix. This tendency to misclassify highredshift (e.g., high-luminosity) X-ray type 1 AGNs as X-ray type 2 AGNs likely accounts for the blue UV-optical continuum of the most luminous X-ray type 2 SED in Figure 6.

\section{XMM SAMPLE: TRENDS IN IRAC COLOR SPACE}

To test for trends in IRAC color space as a function of luminosity, redshift, and AGN type, we plot in Figure 7(a) the IRAC colors of all $X M M$ sources detected in hard X-rays. We then extend the power-law box defined in Section 4 from $\alpha=-3.0$ to $\alpha=1.0$, enclosing $92 \%$ of the XMM sources, and plot in Figure 7(b) the median luminosity, redshift, and $\mathrm{X}$-ray and optical types of the $X M M$ sources in bins of powerlaw slope.

\subsection{Trends in X-Ray Luminosity and Redshift}

A clear trend is seen in X-ray luminosity, with the more luminous X-ray sources displaying redder IRAC colors, as expected (see also Cardamone et al. 2008; Brusa et al. 2009; Eckart et al. 2010; Park et al. 2010; Trump et al. 2011a). While there is no cut in power-law slope that cleanly distinguishes QSOs ( $\left.\log L_{2-10 \mathrm{kev}}\left(\mathrm{erg} \mathrm{s}^{-1}\right)>44\right)$ from less luminous Seyfert galaxies $\left(\log L_{2-10 \mathrm{keV}}\left(\mathrm{erg} \mathrm{s}^{-1}\right)<44\right)$, a cut of $\alpha=-0.78$ best separates the two populations, with equal fractions (72\%) of high-luminosity AGNs falling redward of the cut and lowluminosity AGNs falling blueward of the cut. 

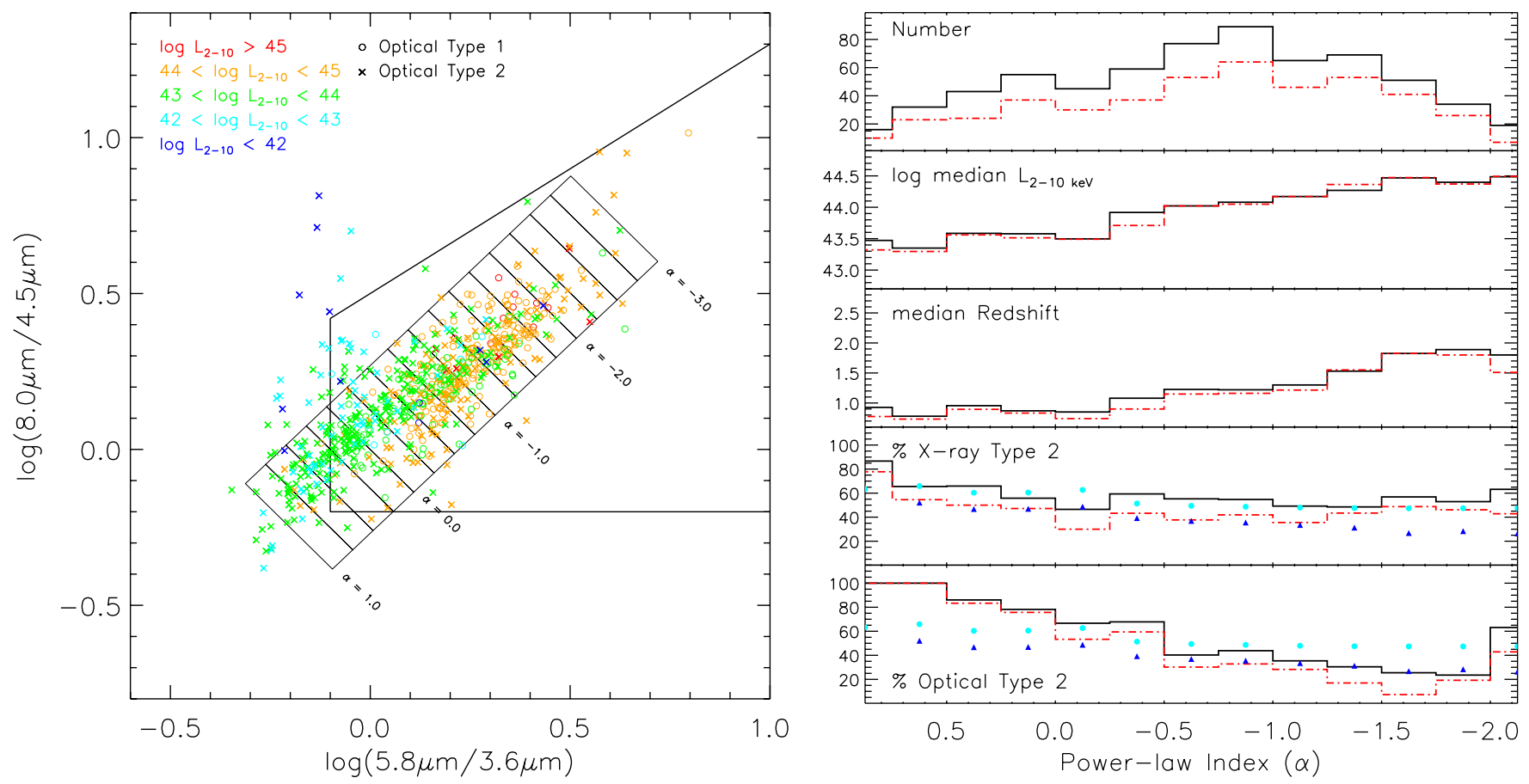

Figure 7. Left panel: IRAC color-color diagram for hard-band-detected $X M M$ sources, as a function of X-ray luminosity and optical type. The power-law box has been extended from $\alpha=1.0$ to -3.0 . Right panel: properties of the XMM sources in each of the bins in $\alpha$ containing at least 10 sources $(\alpha \geqslant-2.25)$. The trends for sources with spectroscopic redshifts are shown by dash-dotted histograms (red in the online journal). The filled circles and triangles give the expected type 2 AGN fractions from Gilli et al. (2007) and Hasinger (2008), respectively, based on the median X-ray luminosity in each bin. AGNs with redder IRAC colors (more negative power-law indices) tend to have higher X-ray luminosities, lie at higher redshift, and be somewhat less obscured.

(A color version of this figure is available in the online journal.)

The median redshift also increases for sources with redder IRAC colors, from $z \sim 1$ at $\alpha=1.0$ to $z \sim 2$ at $\alpha=-2.25$. This correlation is driven primarily by the observed trend in luminosity, as only the most luminous sources (which tend to have redder IRAC colors) can be detected out to high redshift. Between $z=1$ and $z=2$, the SED templates shown in Figure 2 move roughly perpendicularly to the power-law locus, at least for AGN fractions $\leqslant 80 \%$. Only for templates with AGN fractions of $95 \%$ and moderate $A_{\mathrm{V}, \mathrm{AGN}} \sim 2-8$ do we see a trend toward redder IRAC colors between $z=1$ and $z=2$, although this shift of $\Delta \alpha \sim 0.25-0.4$ is too weak to account for the observed correlation between redshift and IRAC color.

\subsection{Trends in AGN Type}

While the X-ray type 2 fraction is essentially independent of $\alpha$, the optical type 2 fraction drops smoothly from $\alpha=1$ to $\alpha \sim-1.6$ before rising again at the reddest IRAC colors. These seemingly inconsistent trends remain, and are even enhanced, if we consider only those sources with spectroscopic redshifts (shown in red).

Because high-luminosity AGNs tend to be less obscured than their low-luminosity counterparts, at least in X-ray-selected samples, we might expect sources with redder IRAC colors to have a lower type 2 fraction (e.g., Ueda et al. 2003; Gilli et al. 2007; Hasinger 2008). We plot in the right panel of Figure 7 the expected type 2 fractions of Gilli et al. (2007) and Hasinger (2008) at the median X-ray luminosity of each bin. While a slight decrease in the type 2 fraction would be expected over the observed range in luminosity, the trend we observe for the optically classified AGNs is much stronger than predicted.

These observed trends, however, reflect the biases and selection effects discussed in Section 5.3. At blue IRAC colors, the excess of low-luminosity, optical type 2 AGNs can be attributed to the population of unobscured narrow-line AGNs in $X M M$-COSMOS (Trump et al. 2011b). Not only are these weak accretors likely to lack a broad-line region, but their anomalously blue IRAC colors also suggest that they, like many lowluminosity, low-accretion-rate radio galaxies, may lack a hot dust torus (Trump et al. 2011b; Ogle et al. 2006). Likewise, the excess of X-ray type 2 AGNs at red IRAC colors can be attributed to the overestimation of X-ray column densities at high redshift (see Section 5.3 and the Appendix).

Given the nature of these biases, an AGN's intrinsic obscuration is best represented by the X-ray classification at low luminosity/redshift, and by the optical classification at high luminosity/redshift. If we therefore assume the X-ray-derived type 2 fraction at blue slopes of $\alpha \gtrsim-0.5$, and the optically derived type 2 fraction at red slopes of $\alpha \lesssim-0.5$, we find a distribution that favors unobscured AGNs at red IRAC colors but whose evolution is not nearly as pronounced as the optical classification alone would suggest. This trend is likely driven primarily by the anti-correlation between luminosity and obscuration, and while this new hybrid estimate of the obscured AGN fraction may display a somewhat steeper evolution than those of Gilli et al. (2007) and Hasinger (2008), a direct comparison is complicated by the scatter in X-ray luminosity in a given power-law bin and our necessary exclusion of sources with ambiguous X-ray types, as well as the expected incompleteness of the $X M M$ sample to the obscured AGNs that are the target of MIR selection.

An optically thick torus could also contribute to the observed trend by masking the hot dust signature from obscured AGNs. As discussed in Section 2, however, the large NIR-MIR anisotropies predicted by models of smooth, geometrically and optically thick tori are not supported by recent observations, 

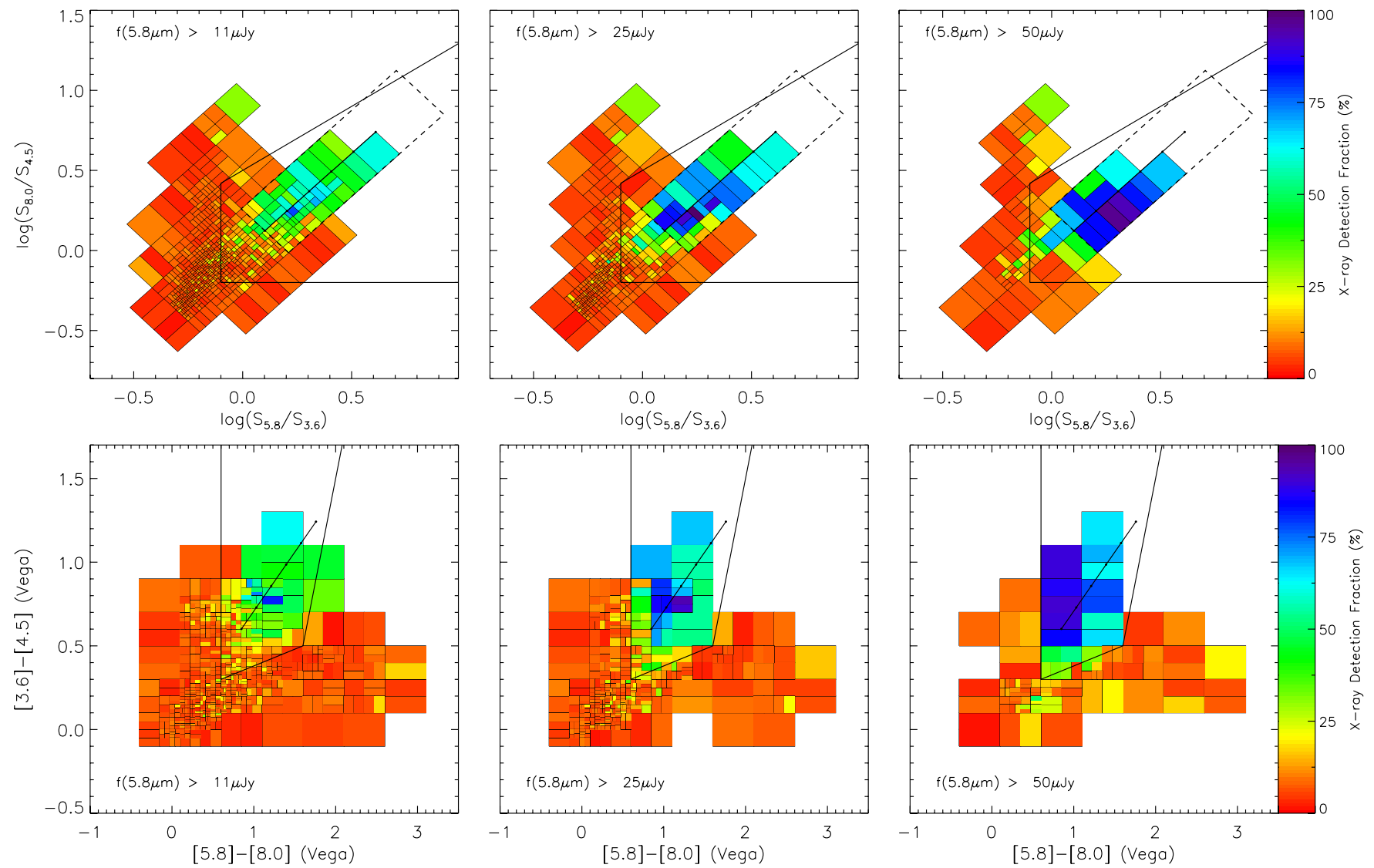

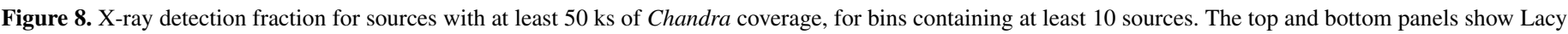

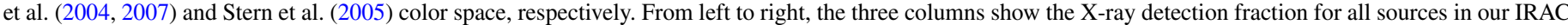

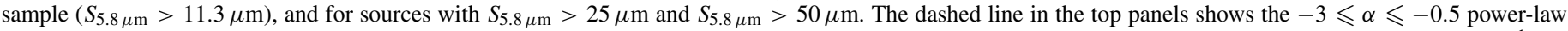

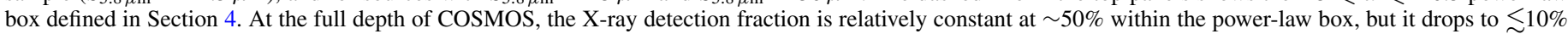
across much of the remainder of IRAC color space, including regions enclosed by the current AGN selection wedges.

(A color version of this figure is available in the online journal.)

particularly when the intrinsic X-ray luminosity is used to normalize the MIR luminosity (Lutz et al. 2004; Gandhi et al. 2009). However, studies that normalize the AGN's luminosity by the radio continuum find a factor-of-a-few difference in MIR luminosity between unobscured and obscured AGNs (Heckman 1995; Haas et al. 2008; Leipski et al. 2010). We return to this issue in Section 9.

\section{IRAC SAMPLE}

While the $X M M$ sample sheds light on the potential completeness of IRAC color selection to AGNs of different luminosities and/or type, $X M M$ sources comprise only $4 \%$ of the full sample of IRAC-COSMOS sources. In the sections that follow, we test our ability to cleanly separate the AGN-dominated IRAC sources from the remainder of the primarily star-formationdominated IRAC population.

\subsection{X-Ray Detection Fraction}

We plot in Figure 8 the Chandra X-ray detection fraction (down to a limiting flux of $5.7 \times 10^{16} \mathrm{erg} \mathrm{s}^{-1}$; Elvis et al. 2009) for the 11324 IRAC sources with at least $50 \mathrm{ks}$ of Chandra coverage, for which the median (mean) X-ray exposure is $148 \mathrm{ks}$ $(128 \mathrm{ks})$. To maximize the resolution in IRAC color space, we divide the IRAC sample into grids containing at least 10 sources. The typical grid size is therefore inversely proportional to the density of IRAC sources in a given region of color space. While the Chandra data set only covers the central $0.9 \mathrm{deg}^{2}$ of the COSMOS field, its increased depth and higher resolution than the larger-area $X M M$ data allow us to probe more deeply the $\mathrm{X}$-ray properties of the IRAC sample.

At the full depth of the IRAC-COSMOS survey, $42 \%$ of the sources in the $\alpha \leqslant-0.5$ power-law box are detected by Chandra, compared to only $5 \%$ of the sources outside the power-law box (see Figure 8). Within the power-law box, the $\mathrm{X}$-ray detection fraction remains relatively constant down to a slope of $\alpha \sim-0.75$. It drops somewhat, however, at the bluest extremes of the power-law box where we might expect contamination from high-redshift galaxies (see Figures 2, 3, and 4). An additional cut in 8.0-4.5 $\mu \mathrm{m}$ color may therefore be warranted, and will be explored in more detail in Section 8.

It is also clear from Figure 8 that at the moderate depth of the IRAC-COSMOS data, significant fractions of the current AGN selection regions of Lacy et al. $(2004,2007)$ and Stern et al. (2005) are characterized by low X-ray detection fractions indistinguishable from the typical values outside of the selection wedges. These regions of color space coincide with the regions where we expect contamination from low- and high-redshift star-forming galaxies, and further motivate the need for new AGN selection criteria.

To illustrate the effects of IRAC depth, we also plot in Figure 8 the X-ray detection fractions for limiting flux densities of 25 and $50 \mu \mathrm{Jy}$ in the $5.8 \mu \mathrm{m}$ band (which effectively limits the detection of red, power-law-like sources). For comparison, the 

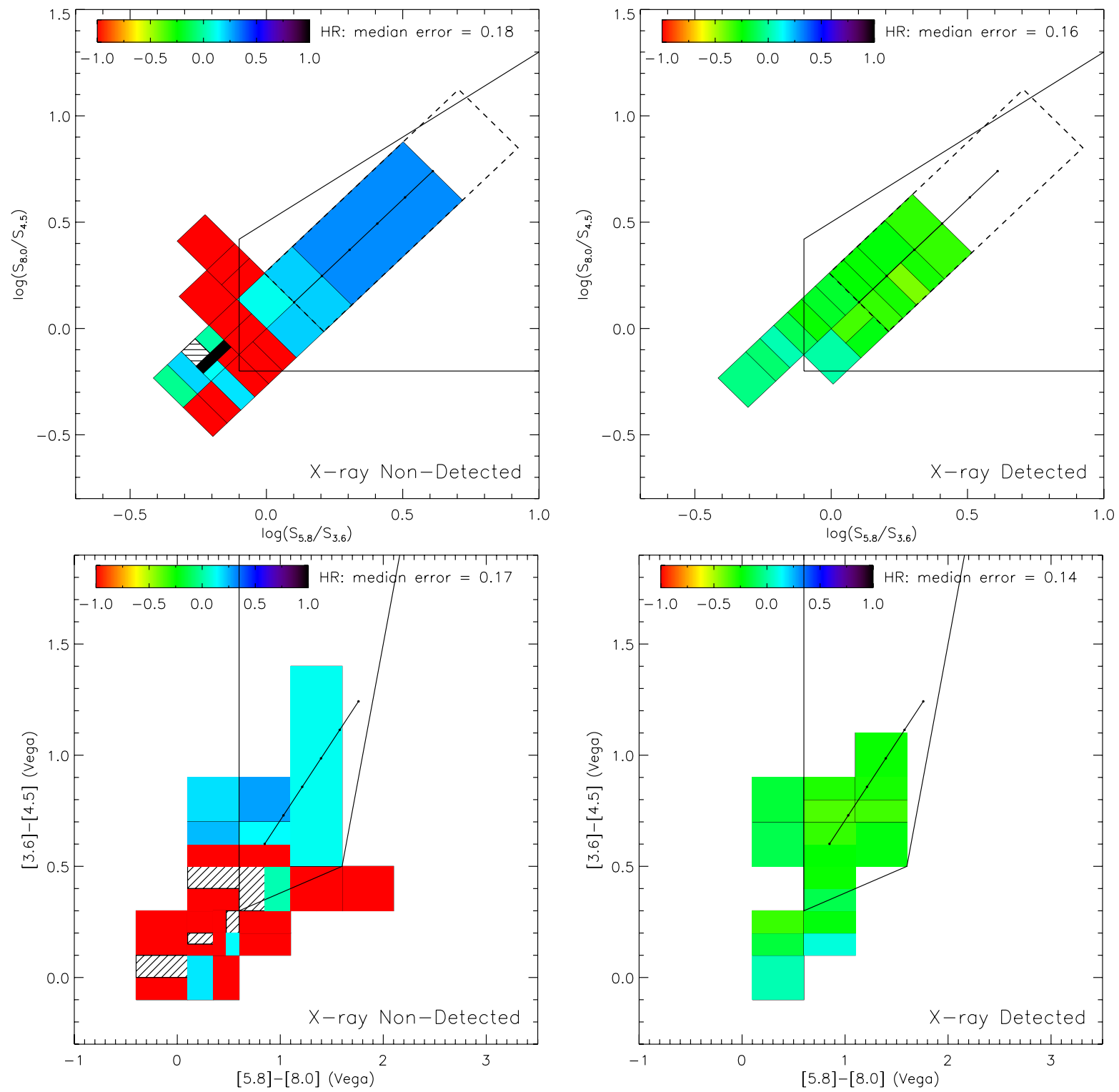

Figure 9. Hardness ratios $(\mathrm{HR}=(H-S) /(H+S))$ of the stacked emission from X-ray-non-detected (left) and X-ray-detected (right) IRAC sources with at least $50 \mathrm{ks}$ of Chandra coverage, where $H$ and $S$ represent the $2-8 \mathrm{keV}$ and $0.5-2 \mathrm{keV}$ ACIS counts, respectively. The top and bottom panels show Lacy et al. (2004, 2007) and Stern et al. (2005) color space, respectively. The minimum number of sources per grid is 200 for the X-ray non-detected sources, and 25 for the X-ray detected sources. White (red in the online journal) and black boxes indicate those grids with a detection in only the soft or hard bands, respectively. Grids detected in neither band are shaded with hashed lines. The dashed line in the top panels shows the power-law box defined in Section 4. The hardest X-ray signal, consistent with emission from obscured to mildly Compton-thick AGNs, comes from sources in the power-law box.

(A color version of this figure is available in the online journal.)

IRAC data used by Lacy et al. (2004) and Stern et al. (2005) to define the current AGN selection regions have limiting $5.8 \mu \mathrm{m}$ depths of $60 \mu \mathrm{Jy}$ and $76 \mu \mathrm{Jy}$, respectively. As the IRAC data become progressively shallower, the X-ray detection fractions rise (to $64 \%$ and $77 \%$ in the power-law box at limiting flux densities of 25 and $50 \mu \mathrm{Jy}$, respectively) and the number of grids in the Lacy et al. $(2004,2007)$ and Stern et al. (2005) AGN selection regions with low, star-formation-like $\mathrm{X}$-ray detection fractions falls. This trend reflects the increase in the AGN fraction with increasing MIR flux density (e.g., Brand et al. 2006; Treister et al. 2006; Donley et al. 2008). While the likelihood of contamination by star-forming galaxies therefore decreases at higher IRAC flux densities, so too does the number of X-ray non-detected AGN candidates in the power-law box, from 570 at the full IRAC depth to 182 and 67 at limiting $5.8 \mu \mathrm{m}$ flux densities of 25 and $50 \mu \mathrm{Jy}$, respectively.

\subsection{X-Ray Stacking}

While the vast majority of IRAC sources lack X-ray counterparts, X-ray stacking can shed light on the nature of the X-ray non-detected population. Using version 3.0beta of the program CSTACK,${ }^{15}$ we stack the X-ray emission for grids in IRAC color space containing at least $200 \mathrm{X}$-ray-undetected sources. We exclude all sources that lie more than $8^{\prime}$ from the Chandra aim-point, and extract source counts within the smaller of either the position-dependent $90 \%$ encircled-energy radius or the $5^{\prime \prime}$ inner background subtraction radius. We then measure the background across the remaining $15^{\prime \prime} \times 15^{\prime \prime}$ image, after masking all known X-ray sources.

We plot in Figure 9 the X-ray hardness ratios, HR, for grids detected to $\geqslant 3 \sigma$ in the soft and/or hard X-ray bands, and plot

\footnotetext{
15 http://cstack.ucsd.edu/cstack/
} 
for comparison the stacked hardness ratios of the X-ray-detected sources $(\mathrm{HR}=(H-S) /(H+S)$, where $H$ and $S$ represent the 2-8 keV and 0.5-2 keV ACIS counts, respectively). While low source densities limit the resolution along the power-law locus, we detect hard X-ray emission from all red $(\alpha \leqslant 0)$ power-law grids in Lacy et al. $(2004,2007)$ color space. In contrast, the majority of grids outside of the power-law box are detected only in soft X-rays, and those with measurable hard X-ray emission lie in regions where we expect to find lower-luminosity AGNs whose hosts dominate their MIR SEDs.

The hardest X-ray signal is observed for sources in the $\alpha<-1.0$ power-law box: $\mathrm{HR}=0.31 \pm 0.13$, where the errors have been derived from the CSTACK bootstrap analysis. For comparison, the stacked emission from X-ray-detected sources in this region of color space is significantly softer: $\mathrm{HR}=-0.31 \pm 0.13$. At the typical photometric redshifts of the X-ray and non-X-ray sources, $z=1.8 \pm 0.7$ and $z=2.1 \pm 1.0$, respectively, these hardness ratios correspond to column densities of $\log N_{\mathrm{H}}\left(\mathrm{cm}^{-2}\right)=22.4 \pm 0.4$ and $\log$ $N_{\mathrm{H}}\left(\mathrm{cm}^{-2}\right)=23.5 \pm 0.4$.

While $61 \%$ of the X-ray sources have spectroscopic redshifts and the remainder have AGN-specific photometric redshifts, only $5 \%$ of the X-ray-non-detected sources in this region of color space have spectroscopic redshifts and their photometric redshifts have been calculated using only normal galaxy templates (Ilbert et al. 2009). To place an independent constraint on the typical redshift of the X-ray-non-detected sources in the $\alpha<-1.0$ power-law box, we turn to their IRAC fluxes, which are on average $3.1 \pm 0.3$ times fainter than those of the X-ray sample. If we assume that this fairly uniform dimming across the four IRAC bands can be attributed solely to redshift effects (i.e., that the X-ray and non-X-ray power-law samples have similar intrinsic MIR luminosities), we estimate a typical redshift of $z=2.7$ and a typical column density of $\log N_{\mathrm{H}}\left(\mathrm{cm}^{-2}\right)=23.7$ for the X-ray-non-detected sources, slightly higher than the values quoted above.

\subsection{X-Ray/MIR Relation}

The observed X-ray to MIR luminosity ratio, a proxy for AGN obscuration, allows us to place an independent constraint on the column densities of the $\alpha \leqslant-1.0$ power-law sources (Lutz et al. 2004; Maiolino et al. 2007; Gandhi et al. 2009; Park et al. 2010). For the X-ray-detected and -non-detected AGNs, we approximate the median $6.7 \mu \mathrm{m}$ rest-frame luminosity from the median power-law slope and observed $8.0 \mu \mathrm{m}$ IRAC flux density, and we calculate the rest-frame 2-10 keV luminosity from the stacked $0.5-2 \mathrm{keV}(1.5-6 \mathrm{keV}$ at $z \sim 2) \mathrm{X}$-ray flux, assuming $\Gamma=1.4$. For the subsample of sources with MIPS $24 \mu \mathrm{m}$ (rest-frame $6.5 \mu \mathrm{m}$ ) counterparts, this extrapolated $6.7 \mu \mathrm{m}$ rest-frame flux density is in excellent agreement with the median observed $24 \mu \mathrm{m}$ flux density.

Using the X-ray/MIR relation of Maiolino et al. (2007), we measure an MIR-derived intrinsic X-ray luminosity of log $L_{2-10 \mathrm{keV}}\left(\mathrm{erg} \mathrm{s}^{-1}\right) \sim 43.8$ for the individually X-ray nondetected $\alpha \leqslant-1.0$ power-law galaxies, $\sim 20$ times higher than observed in the stacked signal. To achieve this degree of hard X-ray suppression, a marginally Compton-thick column density of $\log N_{\mathrm{H}}\left(\mathrm{cm}^{-2}\right) \sim 24$ is required, generally consistent with the HR-derived values above. In comparison, we see no significant offset between the observed and MIR-derived hard $\mathrm{X}$-ray luminosities of the X-ray detected sources in this region of color space $\left(\log L_{2-10 \mathrm{keV}}\left(\mathrm{erg} \mathrm{s}^{-1}\right) \sim 44.1\right)$, as expected for sources with low to moderate $\left(\log N_{\mathrm{H}}\left(\mathrm{cm}^{-2}\right) \leqslant 23\right)$ obscuring columns.

\section{HIGH-REDSHIFT GALAXIES}

The IRAC colors of purely star-forming galaxies generally avoid the power-law region of color space, at least out to $z \lesssim 3$ (see Figure 2). At $z>3$, however, only one IRAC channel samples the red side of the $1.6 \mu \mathrm{m}$ stellar bump, and by $z \sim 5$, the IRAC bands fall entirely on the blue side of this feature. In high-redshift galaxies with older stellar populations and/or measurable dust extinction, this stellar bump has the potential to mimic the power-law emission from AGNs. To determine whether high-redshift star-forming galaxies are an important source of contamination, we investigate below the IRAC colors of high- $z$ sources in COSMOS and in the deeper GOODS fields.

While we could attempt to identify high-redshift candidates purely on the basis of their photometric redshifts, we are primarily interested in sources whose stellar bumps fall redward of the IRAC bands and whose fluxes are faint, precisely those sources for which photometric redshift fitting is most difficult. To constrain the likelihood of contamination by high- $z$ star-forming galaxies, we therefore turn to spectroscopically confirmed and color-selected high- $z$ samples: BzK-selected galaxies (Daddi et al. 2004), distant red galaxies (DRGs; Franx et al. 2003; van Dokkum et al. 2003), Lyman-break galaxies (LBGs; e.g., Steidel et al. 2003), $z>3$ evolved and/or reddened galaxy candidates, and submillimeter galaxies (SMGs). In the IRAC-selected sample of Pérez-González et al. (2008) drawn from the HDF-N, CDF-S, and Lockman Hole fields, $90 \%$ of the IRAC sources at $z=3-3.5$ meet at least one of the BzK, DRG, or LBG criteria, as do $\sim 80 \%$ of the sources at $z=3.5-4$.

\subsection{BzK Galaxies}

The BzK selection technique of Daddi et al. (2004) was designed to identify star-forming (sBzK) and passive (pBzK) galaxies at $1.4 \lesssim z \lesssim 2.5$, although it also identifies luminous AGNs generally removed from BzK-selected samples on the basis of their hard X-ray or MIR power-law emission (see, e.g., Sharp et al. 2002; Daddi et al. 2007b). To constrain the IRAC colors of BzK-selected galaxies in COSMOS, we adopt the COSMOS BzK catalog of McCracken et al. (2010).

We plot in Figures 10(a) and 11(a) the IRAC colors and redshift distributions of the $14 \%$ of sBzK galaxies that meet our IRAC detection criteria. Based on the IRAC color distribution and the relative density of X-ray sources, we divide the IRACdetected sBzK galaxies into two populations. The dominant population (79\%) occupies the same region of IRAC color space used by Huang et al. (2009) to identify $1.5<z<3$ star-forming galaxies: $\log (8.0 \mu \mathrm{m} / 4.5 \mu \mathrm{m})<0.15$ (see Figure 4). Only $11 \%$ of these relatively blue $T_{\mathrm{x}}>50 \mathrm{ks}$ sources have a Chandra counterpart.

The population of $\mathrm{sBzK}$ sources with red IRAC colors $(\log (8.0 \mu \mathrm{m} / 4.5 \mu \mathrm{m}) \geqslant 0.15)$ extends upward along the powerlaw locus and has an X-ray detection fraction of $49 \%$, higher even than the median value in the power-law box (see Section 7.1). While these red sources are therefore strong AGN candidates, Figures 4 and 10 indicate that an additional color cut of $\log (8.0 \mu \mathrm{m} / 4.5 \mu \mathrm{m}) \geqslant 0.15$ should be applied to the AGN selection criteria to prevent contamination from high-redshift star-forming galaxies. This cut, shown in Figure 10, rejects sources in the lower left corner of the powerlaw box, and is slightly bluer than the cut proposed by Coppin 

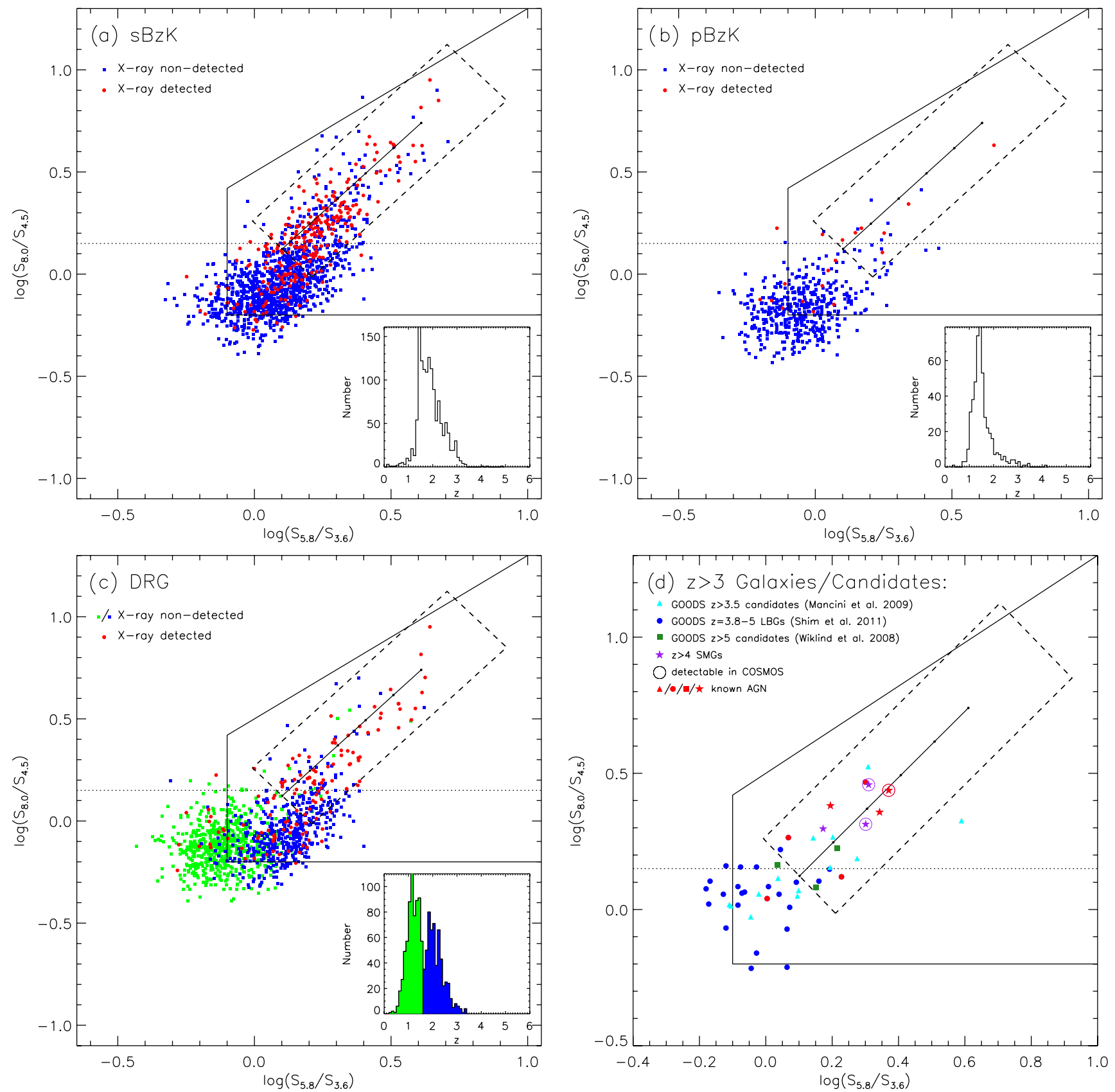

Figure 10. IRAC colors of high- $z$ galaxy populations with at least $50 \mathrm{ks}$ of Chandra coverage, in Lacy et al. (2004, 2007) color space. Black circles (red circles in the online journal) indicate sources with Chandra counterparts, and the inset histograms show the redshift distributions of the high- $z$ populations. The dotted line shows the $\log (8.0 \mu \mathrm{m} / 4.5 \mu \mathrm{m})<0.15$ cut defined in Section 8 to exclude high-redshift star-forming galaxies from the power-law box (dashed line). In panel (d), only the three sources indicated by large circles are bright enough to meet the COSMOS $5 \sigma$ limits in all four IRAC bands. After applying the $8.0 \mu \mathrm{m} / 4.5 \mu \mathrm{m}$ cut indicated by the dotted line, we therefore expect minimal contamination from high-redshift star-forming galaxies.

(A color version of this figure is available in the online journal.)

et al. (2010) to distinguish AGNs and star-formation-dominated SMGs, $\log (8.0 \mu \mathrm{m} / 4.5 \mu \mathrm{m}) \geqslant 0.22$ (see also Yun et al. 2008).

We plot in Figures 10(b) and 11(b) the IRAC colors of the $31 \%$ of pBzK galaxies detected to $\geqslant 5 \sigma$ in the four IRAC bands. Nearly all (97\%) of the pBzK galaxies have IRAC colors blueward of the cut defined above, and only $4 \%$ of these are $\mathrm{X}$-ray sources. Of the few sources with red IRAC colors, 50\% are again X-ray detected.

\subsection{DRGs}

The DRG criterion, $(J-K)_{\mathrm{AB}}>1.37$ or $(J-K)_{\text {Vega }}>2.3$, was designed to select $z=2-3.5$ galaxies with a strong Balmer or $4000 \AA$ break and identifies $77 \%$ of massive galaxies at $z=2-3$ (van Dokkum et al. 2006). Using the UKIRT $J$ and Canada-France-Hawaii Telescope (CFHT) $K_{\mathrm{s}}$ data from Capak et al. (2011) and McCracken et al. (2010), we identify 5000 DRGs in COSMOS, $25 \%$ of which lie above the $\geqslant 5 \sigma$ sensitivity limits in each of the IRAC bands. Their IRAC colors and redshift distribution are shown in Figures 10(c) and 11(c).

While the DRG color cut was originally intended to select sources with a strong Balmer break at $z \sim 2-3.5$, it also recovers dusty star-forming galaxies and AGNs at $z<1.4$ (Conselice et al. 2007; Lane et al. 2007). For clarity, we therefore divide the DRG sample in Figures 10(c) and 11(c) into low- and 

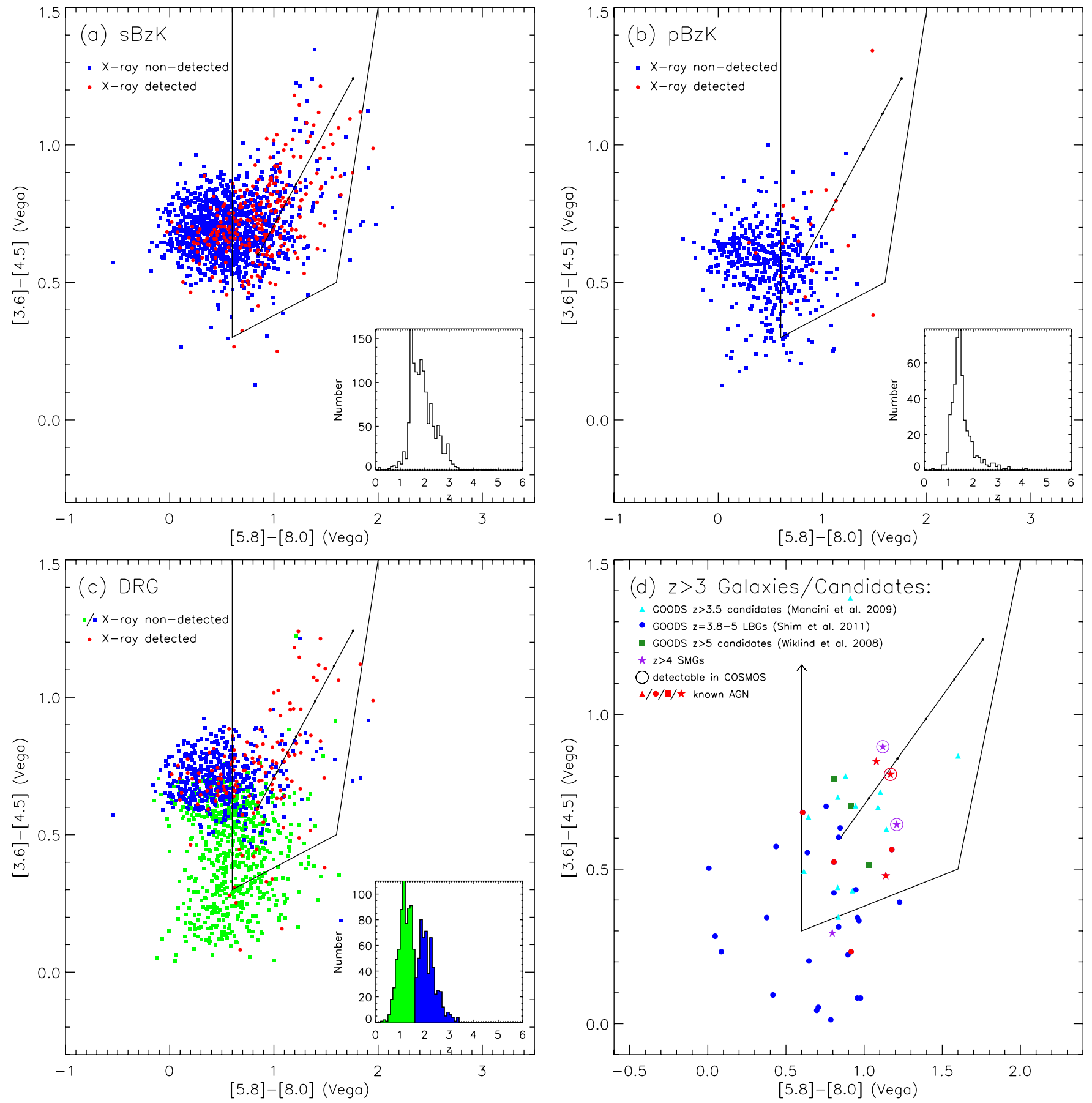

Figure 11. Same as Figure 10, but for the Stern et al. (2005) color space.

(A color version of this figure is available in the online journal.)

high-redshift components, adopting a cut near the minimum in our redshift distribution, $z=1.6$.

The DRGs, like the BzK-selected galaxies, can be divided into two main populations using a cut of $\log (8.0 \mu \mathrm{m} / 4.5 \mu \mathrm{m})=$ 0.15 . The bluer sources, which comprise $91 \%$ of the population, have an X-ray detection fraction of only $6 \%$ and can again be excluded from the AGN selection region using the cut defined above. The red sources extend along the power-law locus and have a far higher X-ray detection fraction of $53 \%$ (or $56 \%$ if we consider only the red sources that lie in the power-law box), indicative of AGN emission. In the deeper GOODS-S field, Papovich et al. (2006) find X-ray or MIR evidence of AGN emission in $25 \%$ of DRGs. In COSMOS, if we take as AGNs all X-ray-detected DRGs as well as all red $(\log (8.0 \mu \mathrm{m} / 4.5 \mu \mathrm{m}) \geqslant 0.15) \mathrm{X}$-ray-non-detected DRGs in the power-law box, we conclude that at least $14 \%$ of the IRACdetected DRGs are AGNs.

\subsection{LBGs}

The Lyman-break dropout technique remains the most successful method of identifying galaxies at $z \sim 3-7$. Of the photometrically selected $U, B, g, V, R$, and $i$ dropout candidates in COSMOS, however, fewer than $2 \%$ are detected at or above the $5 \sigma$ sensitivity limits in all four IRAC bands. As these bright 
sources are also those most likely to be low-redshift interlopers (see, e.g., Reddy et al. 2008), we limit our analysis of LBGs to sources that have been spectroscopically confirmed.

Only three spectroscopically confirmed star-forming $z \geqslant 3$ LBGs in COSMOS are detected to $\geqslant 5 \sigma$ in all four IRAC bands, and two of these are in a close pair with blended IRAC photometry. We therefore turn to the ultra-deep GOODS fields and adopt the sample of 74 spectroscopically confirmed $z=3.8-5.0$ LBGs from Shim et al. (2011), selected to have $\geqslant 5 \sigma$ detections in the 3.6 and $4.5 \mu \mathrm{m}$ IRAC bands. We plot in Figures 10(d) and 11(d) the IRAC colors of the 27 LBGs detected to $\geqslant 3 \sigma$ in all four IRAC bands. As none of the Shim et al. (2011) GOODS sources meet the COSMOS $5 \sigma$ sensitivity cuts in all in four IRAC bands, we do not expect LBGs to be a significant source of contamination at the depth of COSMOS. Nonetheless, their IRAC properties shed light on the potential contamination by high-redshift star-forming galaxies in surveys with deeper IRAC data.

Despite their median redshift of $z=4.2$, the LBGs have relatively blue IRAC colors. Of the 27 LBGs, only 6 fall within the power-law box, and half of these are X-ray detected AGNs. This behavior can be attributed to the requirement that LBGs have a bright UV continuum (which in turn biases the selection toward galaxies with young stellar populations, low extinctions, and thus blue UV-optical rest-frame colors) and to the bright $\mathrm{H} \alpha$ emission observed in $70 \%$ of the Shim et al. (2011) sources, which leads to an excess of $3.6 \mu \mathrm{m}$ emission relative to the bestfit stellar continuum. If no $\mathrm{H} \alpha$ were present, however, the Shim et al. (2011) sources would simply shift to the right in Lacy et al. $(2004,2007)$ color space and continue to be excluded by the $8.0 \mu \mathrm{m} / 4.5 \mu \mathrm{m}$ cut defined above. For a high-redshift galaxy to display red colors in the observed IRAC bands, it must be more heavily reddened or have an older stellar population than the typical LBG.

\subsection{Evolved and Reddened $z>3$ Candidates}

Several attempts have been made to select reddened and/or evolved galaxies at $z>3$ (Yan et al. 2006; Rodighiero et al. 2007; Wiklind et al. 2008; Mancini et al. 2009; Marchesini et al. 2010). The high $24 \mu \mathrm{m}$ detection fraction for these sources, however, points to likely contamination by star-forming galaxies at $z=2-3$ or to a dominant obscured AGN component at high redshift (see, e.g., Dunlop et al. 2007; Marchesini et al. 2010). We therefore plot in Figures 10(d) and 11(d) only the "no-MIPS" $z>3.5$ IRAC-selected and $z>5$ Balmer-break-selected high- $z$ candidates from the GOODS samples of Mancini et al. (2009) and Wiklind et al. (2008), and further restrict the Mancini et al. (2009) sample to those sources lacking a $z \leqslant 3$ redshift solution within the $90 \%$ confidence interval.

None of the high- $z$ evolved and/or reddened galaxy candidates plotted in Figures 10(d) and 11(d) meet the COSMOS $5 \sigma$ sensitivity limits in all four IRAC bands, so we again expect little to no contamination from such galaxies in COSMOS. Furthermore, only 5 of the $13 z>3.5$ candidates from Mancini et al. (2009) lie within the power-law box, primarily at colors blueward of $\alpha \sim-1.0$. (The reddest Mancini et al. 2009 source in the power-law box is the $z_{\text {phot }}=3.93$ SMG GN1200.5/AzGN06.) Likewise, two of the three $z \geqslant 5$ Balmer-break candidates of Wiklind et al. (2008) fall in the power-law box, though again at $\alpha \geqslant-1.0$. The relatively blue continua of these galaxies, compared to the redshifted tracks of local templates, can likely be attributed to the bluer UV continua and lower dust extinctions of high-redshift galaxies (Bouwens et al. 2009). Even in the deep GOODS field, we therefore expect only minimal contamination from typical high-redshift galaxies, and only in the bluest regions of the power-law box.

\subsection{SMGs}

While dust extinction appears to be less prevalent in the early universe, heavily dust obscured luminous star-forming galaxies have been selected as SMGs out to $z \sim 5$ (Capak et al. 2008; Schinnerer et al. 2008; Daddi et al. 2009a, 2009b; Coppin et al. 2009; Knudsen et al. 2010; Riechers et al. 2010; Smolčić et al. 2011). At moderate redshifts of $1.4<z<2.6$, more than $80 \%$ of bright radio-selected SMGs meet one or more of the BzK, DRG, or LBG (e.g., BM/BX) criteria (Reddy et al. 2005). Their IRAC colors are therefore coincident with the BzK and DRG star-forming populations shown in Figures 10(a), 11(a), 10(b), and 11(b) (see also Figure 4). At $z \gtrsim 4$, however, UV-NIR color cuts are unlikely to identify the dust-obscured and IR-luminous SMGs most likely to enter the AGN selection region (though see Mancini et al. 2009).

In COSMOS, six spectroscopically confirmed $z \gtrsim 4$ SMGs have been identified over $0.2 \mathrm{deg}^{2}$. Of these, only AzTEC5 (Marchesini et al. 2010; P. Capak et al., in preparation) is detected to $\geqslant 5 \sigma$ in all four IRAC bands, although AzTEC1 (Smolčić et al. 2011) falls just below our cuts. We plot in Figures $10(d)$ and 11(d) the IRAC colors of these two high- $z$ SMGs, as well as the four (of seven) additional spectroscopically confirmed $z>4$ SMGs with high- $\sigma$ IRAC counterparts: the GOODS-N sources GN20 and GN20.1 (Daddi et al. 2009b), the EGS source LESS J033229.4 (Coppin et al. 2009; Gilli et al. 2011), and the A2218 source SMM J163555.5 (Knudsen et al. 2010).

All of the $z \geqslant 4$ SMGs fall in the power-law box at $-1<\alpha<-2$, although only three are bright enough to be included (or nearly included) in our IRAC-COSMOS sample. At least half of the $z \geqslant 4$ SMGs, however, are known AGNs (GN20.2 is a radio-bright AGN (Daddi et al. 2009b), LESS J033229.4 is a Compton-thick AGN detected in the $4 \mathrm{Ms}$ Chandra data (Gilli et al. 2011), and the IRS spectrum of GN20 indicates a considerable AGN contribution to the rest-frame $6 \mu \mathrm{m}$ emission (D. Riechers et al., in preparation)), and all but one have a bright MIPS counterpart that points to a likely AGN contribution. We therefore expect little to no contamination from predominantly star-forming SMGs at moderate to high redshifts.

\section{REVISED IRAC CRITERIA}

We take as a starting point for the revised IRAC selection criteria the $\alpha \leqslant-0.5$ power-law box defined in Section 4 . Not only does this box enclose the vast majority of sources that would be identified as power-law galaxies (Alonso-Herrero et al. 2006; Donley et al. 2007; Park et al. 2010), but it also tightly encloses the templates of AGN-dominated sources (see Figure 2) and the region of color space with a high $(\sim 50 \%)$ X-ray detection fraction (see Figure 8).

To prevent contamination from high-redshift $(z \geqslant 2)$ galaxies bright enough to be included in the IRAC-COSMOS sample, we then impose a cut of $\log (8.0 \mu \mathrm{m} / 4.5 \mu \mathrm{m}) \geqslant 0.15$, defined in Section 8. While this high $-z$ cut removes the majority of grids in the power-law box with low X-ray detection fractions (see Figure 8), the leftmost corner of the power-law box also has a lower-than-average X-ray detection fraction, presumably due to contamination by low-redshift star-forming galaxies. To exclude these sources, we impose a vertical cut that coincides 

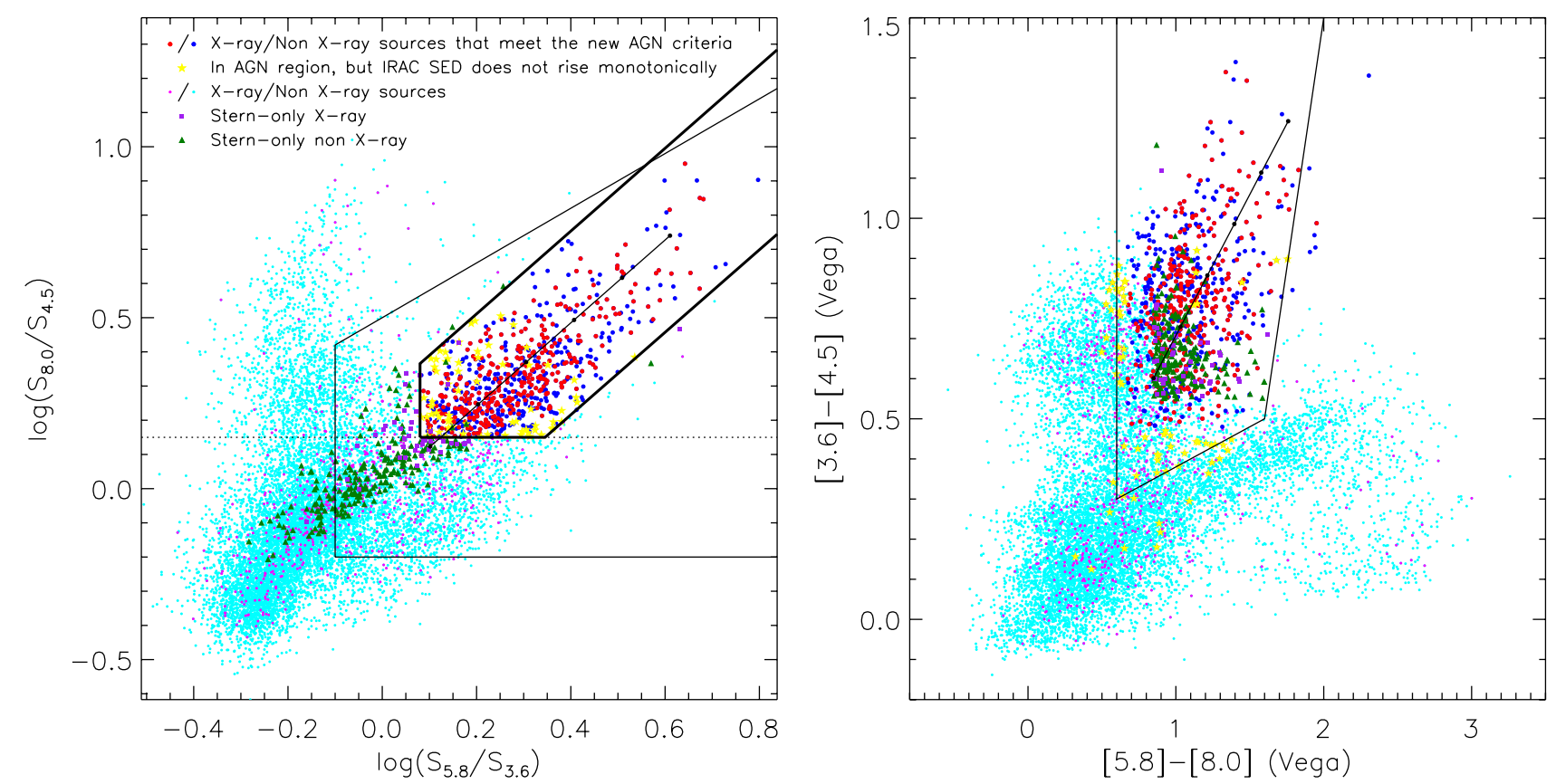

Figure 12. New AGN selection criteria (thick solid lines). The full IRAC-COSMOS sample with $T_{x} \geqslant 50 \mathrm{ks}$ are plotted as small points, and sources that meet our new criteria are shown by large filled circles. Sources plotted in black (or red/magenta in the online journal) have X-ray counterparts, and sources plotted in gray (or blue/cyan in the online journal) do not. Large (yellow) stars represent sources that lie within the revised wedge in Lacy et al. (2004, 2007) color space but that are excluded from our final selection because their IRAC SEDs do not rise monotonically. The (purple) squares and (green) triangles represent the X-ray and non-X-ray sources, respectively, that fall well within the Stern et al. (2005) AGN selection region but that are excluded by our new AGN selection criteria (see Section 9.2.3).

(A color version of this figure is available in the online journal.)

with the intersection of the $\alpha \leqslant-0.5$ power-law box and the high $-z$ cut discussed above: $\log (5.8 \mu \mathrm{m} / 3.6 \mu \mathrm{m}) \geqslant 0.08$.

We plot in Figure 12 the IRAC colors of the sources that meet these criteria. While all of the new AGN candidates fall by definition within the original Lacy et al. $(2004,2007)$ AGN selection wedge, 9\% have IRAC SEDs that do not rise monotonically, but that decrease between 3.6 and $4.5 \mu \mathrm{m}$ or 5.8 and $8.0 \mu \mathrm{m}$ (or, in rare cases, between 4.5 and $5.8 \mu \mathrm{m}$ ), placing them on the outskirts of the Stern et al. (2005) AGN selection region (of these, $38 \%$ formally fall outside of the Stern et al. 2005 AGN wedge). This behavior is rare among the XMM AGNs in our selection region, $97 \%$ of which have monotonically rising IRAC SEDs (a fraction that rises to $99.6 \%$ if we consider only luminous QSOs with $\left.\log L_{2-10 \mathrm{kev}}\left(\mathrm{erg} \mathrm{s}^{-1}\right) \geqslant 44\right)$, and may be due to the $1.6 \mu \mathrm{m}$ stellar bump passing through the IRAC bandpasses. This interpretation is consistent with the median redshifts, $z=0.7$ and $z=2.2$, and low $T_{\mathrm{x}}>50 \mathrm{ks} \mathrm{X}$-ray detection fractions, $17 \%$ and $30 \%$, of the sources with blue 3.6/4.5 $\mu \mathrm{m}$ and 5.8/8.0 $\mu \mathrm{m}$ colors, respectively. We therefore exclude from our final selection criteria all sources with nonmonotonically rising IRAC SEDs, plotted as yellow stars in Figure 12. Of the low-redshift sources previously excluded by the vertical cut in Lacy et al. $(2004,2007)$ color space, $81 \%$ would also be excluded by this criterion.

The final revised AGN selection criteria are as follows, where $\wedge$ is the logical "AND" operator:

$$
\begin{gathered}
x=\log _{10}\left(\frac{f_{5.8 \mu \mathrm{m}}}{f_{3.6 \mu \mathrm{m}}}\right), \quad y=\log _{10}\left(\frac{f_{8.0 \mu \mathrm{m}}}{f_{4.5 \mu \mathrm{m}}}\right) \\
x \geqslant 0.08 \wedge y \geqslant 0.15 \\
\wedge y \geqslant(1.21 \times x)-0.27 \wedge y \leqslant(1.21 \times x)+0.27 \\
\wedge f_{4.5 \mu \mathrm{m}}>f_{3.6 \mu \mathrm{m}} \wedge f_{5.8 \mu \mathrm{m}}>f_{4.5 \mu \mathrm{m}} \wedge f_{8.0 \mu \mathrm{m}}>f_{5.8 \mu \mathrm{m}}
\end{gathered}
$$

These new criteria identify 1506 AGN candidates in COSMOS, only $38 \%$ of which have $X M M$ or Chandra counterparts. In regions of deep Chandra coverage $\left(T_{\mathrm{x}}=50-160 \mathrm{ks}\right)$, the X-ray detection fraction is $52 \%$.

Repeating the X-ray stacking analysis for the final AGN candidate sample confirms the results of Section 7.2. The X-raydetected AGNs that meet our IRAC criteria lie at $z=1.7 \pm 0.8$ and have typical stacked hardness ratios and column densities of $\mathrm{HR}=-0.30 \pm 0.11$ and $\log N_{\mathrm{H}}\left(\mathrm{cm}^{-2}\right)=22.4 \pm 0.4$. In comparison, the $\mathrm{X}$-ray-non-detected $\mathrm{AGN}$ candidates with $T_{\mathrm{x}}>50 \mathrm{ks}$ (median $T_{\mathrm{x}}=125 \mathrm{ks}$ ) have slightly higher photometric redshifts: $z_{\text {phot }}=2.2 \pm 0.9$. While this population lacks individual X-ray counterparts, X-ray stacking leads to $\sim 6 \sigma$ detections in both the soft and hard X-ray bands with $\mathrm{HR}=0.29 \pm 0.13$ or $\log N_{\mathrm{H}}\left(\mathrm{cm}^{-2}\right)=23.5 \pm 0.4$. IRAC selection therefore appears to successfully recover large samples of both unobscured to moderately obscured X-ray-detected AGNs, as well as heavily obscured, high-redshift AGNs missed by deep $\mathrm{X}$-ray surveys.

\subsection{Reliability}

The criteria defined above have been designed to reject the majority of low- and high-redshift star-forming contaminants that enter the Lacy et al. (2004, 2007) and Stern et al. (2005) AGN selection wedges (see Sections 2, 7, 8, and 9). While approximately half of the AGN candidates selected by these criteria are not individually detected in deep $\left(T_{\mathrm{x}}=50-160 \mathrm{ks}\right)$ Chandra data, their stacked X-rays are consistent with emission from heavily obscured AGNs. However, $32 \%$ of the $T_{\mathrm{x}}>50 \mathrm{ks}$ X-ray-undetected IR AGN candidates have $z_{\text {phot }} \geqslant 2.7$, the redshift at which the templates of purely star-forming local LIRGS and ULIRGS from Rieke et al. (2009) begin to enter our new selection region. To determine if these sources are starforming contaminants, we repeat the X-ray stacking analysis for 
three equally populated redshift bins: $z=0-1.8, z=1.8-2.7$, and $z=2.7-4.8$.

The stacked emission from the $z>2.7$ IR AGN candidates is detected to $3.9 \sigma$ in the $0.5-2.0 \mathrm{keV}$ band and to $4.0 \sigma$ in the $2-8 \mathrm{keV}$ band with an observed hardness ratio of $\mathrm{HR}=0.16 \pm 0.18\left(\right.$ or $\log N_{\mathrm{H}}\left(\mathrm{cm}^{-2}\right)=23.6 \pm 0.2$ at the mean redshift of $z=3.2$ ). As the effective X-ray photon index, $\Gamma_{\text {eff }}=0.67_{-0.36}^{+0.35}$, is harder than even the hardest starforming galaxy $\left(\Gamma_{\mathrm{eff}}=1-2\right.$; e.g., Lehmer et al. 2008), a significant fraction of these sources must be obscured AGNs. ${ }^{16}$ For comparison, the low-redshift bin is detected to $2.4 \sigma$ in the soft band and to $4.1 \sigma$ in the hard band with $\mathrm{HR}=0.50 \pm 0.23$ or $\Gamma_{\text {eff }}=-0.04_{-0.55}^{+0.51}\left(\log N_{\mathrm{H}}\left(\mathrm{cm}^{-2}\right)=23.2 \pm 0.4\right.$ at $\left.z=1.2\right)$, and the medium redshift bin is detected to $3.2 \sigma$ in the soft band and to $2.7 \sigma$ in the hard band with $\mathrm{HR}=0.26 \pm 0.27$ or $\Gamma_{\text {eff }}=0.49_{-0.58}^{+0.51}\left(\log N_{\mathrm{H}}\left(\mathrm{cm}^{-2}\right)=23.5 \pm 0.3\right.$ at $\left.z=2.2\right)$.

Many of the high- $z$ AGN candidates, however, show a clear infrared excess relative to even the reddest star-forming template. To better isolate the sources most likely to be starforming contaminants, we develop the following empirical criteria to identify high- $z$ sources whose IRAC photometry can plausibly be fit by the Rieke et al. (2009) star-forming templates:

$$
\begin{gathered}
\log _{10}\left(\frac{f_{8.0 \mu \mathrm{m}}}{f_{3.6 \mu \mathrm{m}}}\right)< \begin{cases}0.39 \times z-0.69 & \text { if } \mathrm{z}=2.7-3.1 \\
0.18 \times z-0.04 & \text { if } \mathrm{z}=3.1-4.2 \\
0.06 \times z+0.47 & \text { if } \mathrm{z}=4.2-5.0\end{cases} \\
\text { and } \frac{f_{5.8 \mu \mathrm{m} / f_{3.6 \mu \mathrm{m}}} \geqslant 0.95}{f_{8.0 \mu \mathrm{m} / f_{4.5 \mu \mathrm{m}}}}
\end{gathered}
$$

The first criterion identifies sources whose 8.0-3.6 $\mu \mathrm{m}$ flux ratio is not significantly redder than the reddest Rieke et al. (2009) LIRG/ULIRG template at a given redshift, and the second criteria ensures that the curvature of the IRAC photometry is roughly consistent with the redshifted $1.6 \mu \mathrm{m}$ stellar bump. Approximately $50 \%$ of the $z>2.7$ X-ray-non-detected IR AGN candidates (or $15 \%$ of the full sample of X-ray-non-detected AGNs) meet these criteria and therefore have IRAC photometry potentially consistent with star formation at their assumed redshift (though nearly all show an infrared excess relative to the bluest of the Rieke et al. 2009 templates). X-ray stacking of this subsample, however, again returns significant $(>3 \sigma)$ detections in both the soft and hard X-ray bands with $\mathrm{HR}=0.33 \pm 0.22$ or $\Gamma_{\text {eff }}=0.35_{-0.50}^{+0.44}$, indicating that any contamination by highredshift star-forming galaxies is minimal. Instead, these sources appear to be either high-redshift AGNs whose underlying hosts are bluer than the reddest Rieke et al. (2009) template, or lower redshift AGNs whose photometric redshifts have been artificially inflated by the fact that they were estimated using only star-forming templates, which are consistent with red, power-law-like IRAC emission only at high- $z$. Interestingly, we do not detect a significant stacked signal for the $50 \%$ of $z>2.7$ AGN candidates with extremely red IRAC colors, perhaps because these sources lie at genuinely higher redshifts or are more heavily obscured.

\subsection{Completeness}

To quantify the completeness of the new selection criteria, we plot in Figure 13(a) the fraction of hard X-ray- and

\footnotetext{
16 We use v4.2 of the Portable, Interactive Multi-Mission Simulator (PIMMS) to estimate $\Gamma_{\text {eff }}$ using the Galactic column density of $2.7 \times 10^{20} \mathrm{~cm}^{-2}$ (Elvis et al. 2009) and the Chandra Cycle 8 effective area curves.
}

IRAC-detected XMM and Chandra sources that fall in our new selection region as a function of their intrinsic $2-10 \mathrm{keV}$ luminosities. The completeness of our new selection criteria is a strong function of AGN luminosity, as expected. At log $L_{\mathrm{X}}\left(\mathrm{erg} \mathrm{s}^{-1}\right)<43$, fewer than $20 \%$ of AGNs meet our criteria due to dilution of the AGN continuum by the host galaxy and/or to the disappearance of the hot dust torus at low Eddington accretion rate (Trump et al. 2011a). At QSO luminosities of $\log L_{\mathrm{x}}\left(\mathrm{erg} \mathrm{s}^{-1}\right) \geqslant 44$, however, $75 \%$ of the XMM AGNs and $68 \%$ of the Chandra AGNs fall in the revised IRAC selection region. ${ }^{17}$

\subsubsection{Nature of the QSOs Missed by Our Selection Criteria}

We plot in Figure 13(b) the best fits to the median SEDs of the $X M M$ QSOs (e.g., $\left.\log L_{\mathrm{x}}\left(\mathrm{erg} \mathrm{s}^{-1}\right) \geqslant 44\right)$ that fall inside and outside of our selection region, calculated as described in Section 5.3. The XMM-selected QSOs that meet our criteria have a median redshift of $z=1.8$, a median luminosity of $\log L_{\mathrm{X}}\left(\mathrm{erg} \mathrm{s}^{-1}\right)=44.4$, a median column density of $\log$ $N_{\mathrm{H}}\left(\mathrm{cm}^{-2}\right)=22.3$ (for the $82 \%$ of sources with a measurable column), and X-ray and optical type 2 fractions of $53 \%$ and $28 \%$, respectively. The type 1 and type 2 SEDs are remarkably similar at $\lambda \gtrsim 1 \mu \mathrm{m}$, and they show no sign of host galaxy emission.

In comparison, the $X M M$-selected QSOs (again defined to have $\left.\log L_{\mathrm{X}}\left(\mathrm{erg} \mathrm{s}^{-1}\right) \geqslant 44\right)$ that fall outside of our selection region have a median redshift of $z=1.6$, a median luminosity of $\log L_{\mathrm{X}}\left(\mathrm{erg} \mathrm{s}^{-1}\right)=44.2$, a median column density of $\log$ $N_{\mathrm{H}}\left(\mathrm{cm}^{-2}\right)=22.6$ (for the $88 \%$ of sources with a measurable column), and X-ray and optical type 2 fractions of $74 \%$ and $54 \%$, respectively. Their SEDs are characterized by slightly redder UV-optical continua and by noticeable $1.6 \mu \mathrm{m}$ stellar bumps, even in the case of the type 1 QSOs. The QSOs missed by IRAC selection therefore appear to be somewhat more heavily obscured, lower luminosity AGNs whose host galaxies contribute a larger relative fraction of their optical-NIR flux.

\subsubsection{Completeness to Heavily Obscured AGNs}

To better constrain our completeness to heavily obscured AGNs, we turn to the luminous $\left(f_{24} \geqslant 700 \mu \mathrm{Jy}\right)$ dustobscured galaxies (DOGs; Dey et al. 2008) of Donley et al. (2010), selected on the basis of their high $24 \mu \mathrm{m}$ to $R$-band flux ratios. All but one of these AGN-dominated sources at $z \sim 2$ have QSO-like luminosities (e.g., $\log L_{\mathrm{x}}\left(\mathrm{erg} \mathrm{s}^{-1}\right) \geqslant 44$ ), and as many as $80 \%$ may be Compton-thick. Eight of the eleven QSO-luminosity DOGs with good IRAC data meet our new IRAC criteria, with one additional source falling just beyond our selection region.

The two DOGs that fall well outside of our selection region (IRBG10 and IRBG13) are the brightest radio sources in the sample, and both show a distinct curvature in their IRAC SEDs, with an excess of 3.6 and $8.0 \mu \mathrm{m}$ emission and/or a deficit of 4.5 and $5.8 \mu \mathrm{m}$ emission (see Figure 1 of Donley et al. 2010). Haas et al. (2008) and Leipski et al. (2010) observe similar SEDs among their sample of $z>1178 \mathrm{MHz}$ selected double-lobed radio galaxies (e.g., radio-selected type 2 AGNs), which they attribute to the combined effects of AGN obscuration and host-galaxy emission. It is perhaps not surprising, then, that while the new IRAC selection criteria recover all of

\footnotetext{
17 See Richards et al. (2009) and Assef et al. (2010) for a discussion of the effect of $H \alpha$ emission on the selection of luminous type 1 QSOs at higher redshift $(z \sim 4.5)$.
} 

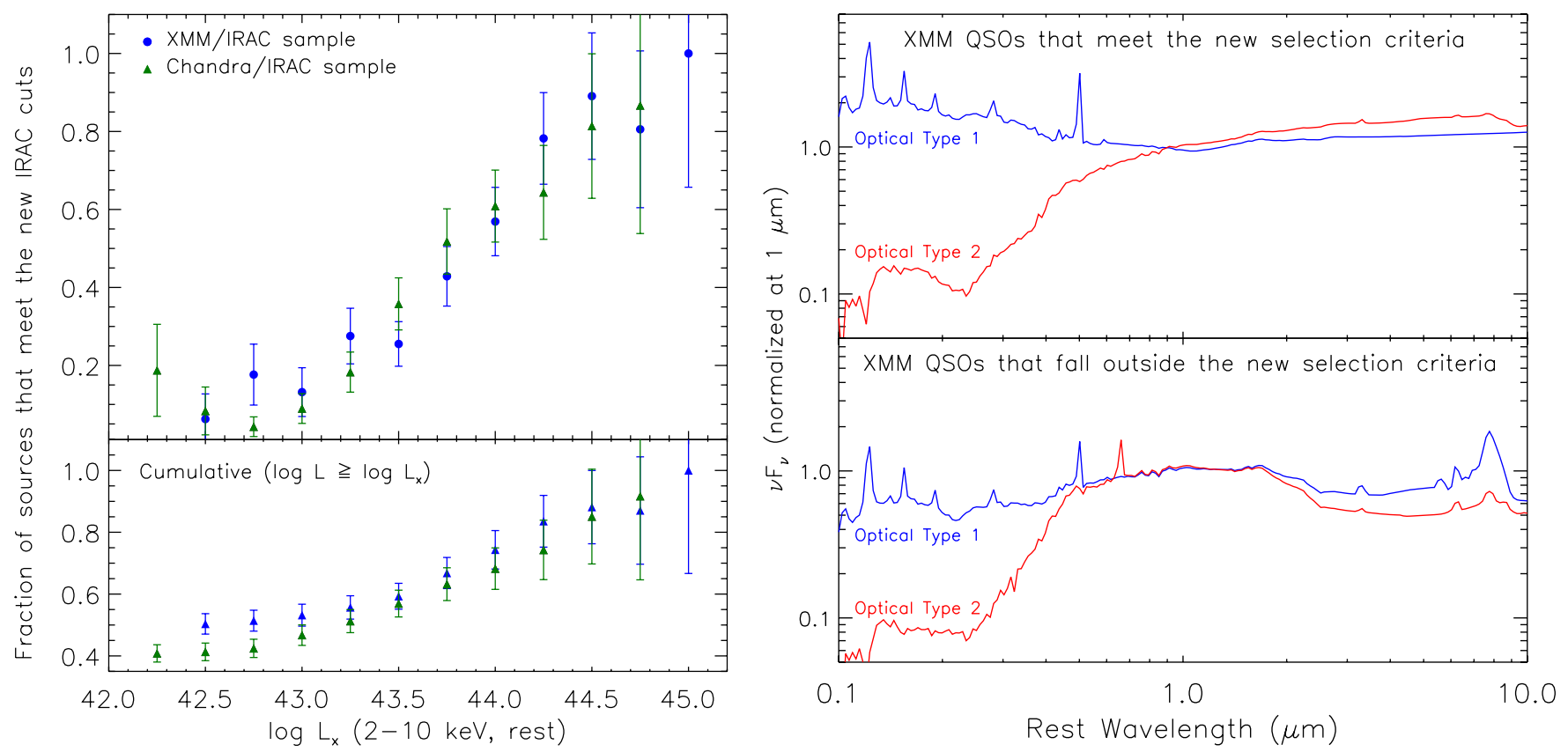

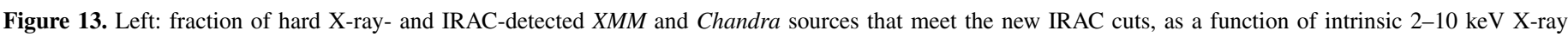

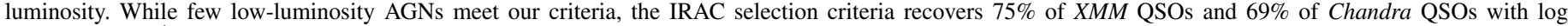

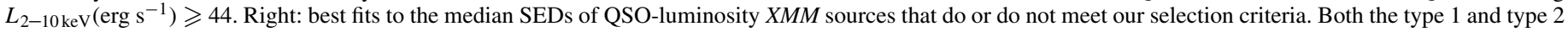

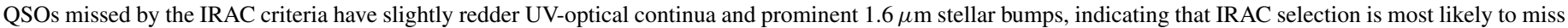
AGNs with luminous hosts, particularly when the AGN emission is itself obscured.

(A color version of this figure is available in the online journal.)

the type 1 3CRR radio quasars of Haas et al. (2008), they recover only $33 \%$ of the $z=1.3 \pm 0.2$ narrow-line (e.g., type 2) 3CRR radio galaxies, whose low-frequency selection and complete optical identification should result in an obscurationindependent sample of luminous radio-loud AGNs. ${ }^{18}$

To extend this test to higher redshift, we adopt the Spitzer high $z$ radio galaxy (SHzRG) sample of Seymour et al. (2007) and De Breuck et al. (2010). Of the $z=2.2 \pm 0.8$ type 2 SHzRGs with good IRAC photometry (approximately half of the full SHzRG sample), $61 \%$ lie within our AGN selection region. The AGNs that are missed tend to have the highest star-forming contributions to their $1.6 \mu \mathrm{m}$ rest-frame flux and the lowest radio core fractions, indicating that they are preferentially viewed in edge-on, potentially heavily obscured orientations. While the SHzRG and $z>1$ 3CRR radio galaxy samples lie at different redshifts, they have similar rest-frame radio luminosities. The higher recovery fraction of the SHzRGs is therefore likely due to the more heterogeneous selection of this sample, whose larger range of core fractions (e.g., orientations) relative to the 3CRR sample suggests a bias toward less heavily obscured radio galaxies.

The X-ray stacking results from Sections 7 and 9 and the Donley et al. (2010) DOG sample illustrate that a large fraction of IR-luminous, potentially Compton-thick AGNs can be recovered by our IRAC criteria. Nonetheless, many to most radio-selected type 2 luminous AGNs lie outside of our selection region, depending on how the radio sample is selected. This difference may stem from the more luminous host galaxies of radio-loud AGNs (e.g., Lacy et al. 2000). While obscuration in AGNs far more luminous than their hosts will lead to a

\footnotetext{
18 The $z>13$ CRR radio galaxies discussed here lie at higher redshifts and have higher radio luminosities $\left(v L_{v}(178 \mathrm{MHz}\right.$, rest $\left.)>10^{44} \mathrm{erg} \mathrm{s}^{-1}\right)$ than the intrinsically MIR-weak and LINER-like narrow-line radio galaxies of Ogle et al. (2006), which may lack a hot dust torus.
}

reddening of the IRAC SED similar to that seen in the top panel of Figure 13(b), obscuration in lower-luminosity AGNs or those with particularly luminous early-type hosts will more quickly reveal the stellar continuum, resulting in the curved NIR-MIR SEDs shown in the bottom panel of Figure 13(b) and observed by Donley et al. (2010), Haas et al. (2008), and Leipski et al. (2010). IRAC selection will therefore preferentially miss AGNs with more luminous hosts, particularly when the AGN emission is itself obscured.

\subsubsection{Stern et al. (2005) Selected Sources}

By definition, the AGN candidates occupy a well-defined region of Lacy et al. $(2004,2007)$ IRAC color space (though we select only $17 \%$ of the sources that fall in the Lacy et al. 2004, 2007 wedge). While nearly all of our new AGN candidates also lie within the Stern et al. (2005) AGN selection wedge, they comprise only $28 \%$ of the sources in the Stern et al. (2005) wedge and cannot be cleanly separated from the remaining galaxies in this representation of IRAC color space. To investigate the properties of the sources that do not meet our selection criteria, but that lie well within the Stern et al. (2005) wedge in regions populated by our AGN candidates, we plot in Figure 12 the IRAC colors of all $T_{\mathrm{x}} \geqslant 50 \mathrm{ks}$ sources that do not meet our criteria but that have [3.6]-[4.5] (Vega) $>0.55$ and [5.8]-[8.0] (Vega) > 0.85.

This "Stern-only" sample, which comprises $33 \%$ of the sources in this region of IRAC color space (and 17\% of the Stern et al. (2005) selected sources that do not meet our new criteria), has a median redshift of $z=1.5$, an X-ray detection fraction of only $14 \%$, and median 5.8 and $8.0 \mu \mathrm{m}$ flux densities $\left(f_{5.8 \mu \mathrm{m}}=15 \mu \mathrm{Jy}, f_{8.0 \mu \mathrm{m}}=23 \mu \mathrm{Jy}\right)$ that are two to three times fainter than our AGN candidates in this region of color space (though no such offset is seen at 3.6 and $4.5 \mu \mathrm{m}$ ). 


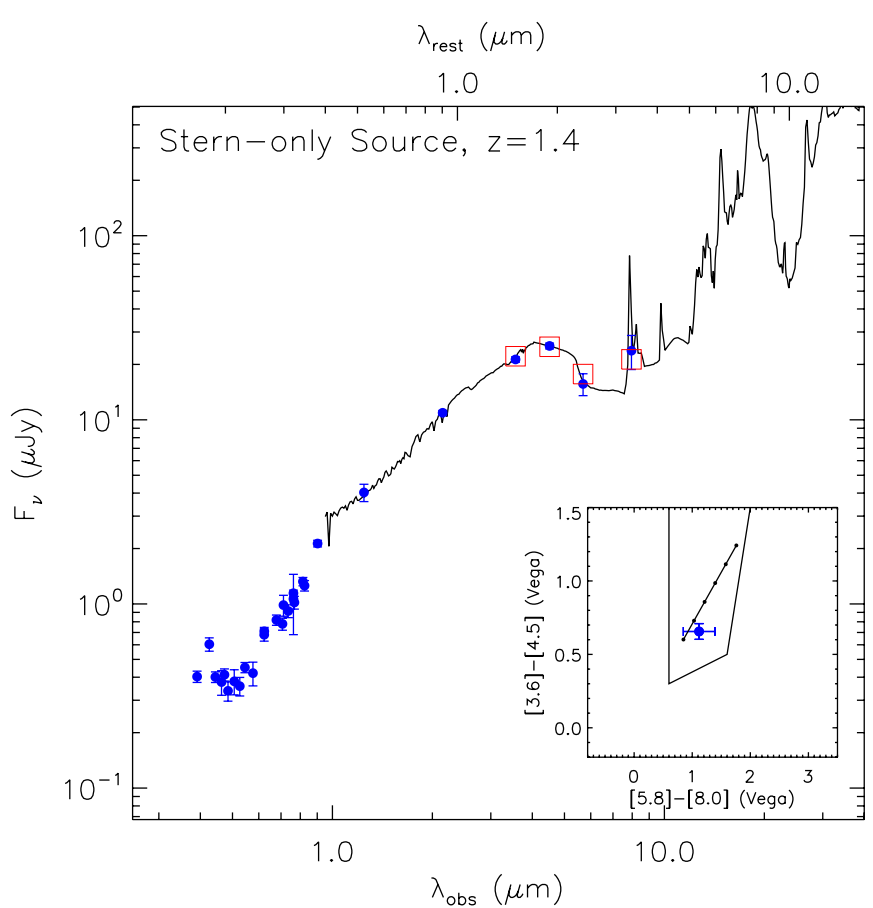

Figure 14. SED of a "Stern-only" source at $z_{\text {phot }}=1.4$, with the Rieke et al. (2009) template of the purely star-forming ULIRG IRAS 12112+0305 overplotted (after scaling to match the observed $4.5 \mu \mathrm{m}$ flux density). Circles give the source photometry, and squares give the photometry of the template convolved with the IRAC bandpasses. The apparently red color of this source in Stern et al. (2005) color space can be attributed solely to star-forming features (e.g., the $1.6 \mu \mathrm{m}$ stellar bump and the $3.3 \mu \mathrm{m}$ PAH emission feature).

(A color version of this figure is available in the online journal.)

While these sources have red 3.6-4.5 $\mu \mathrm{m}$ and 5.8-8.0 $\mu \mathrm{m}$ colors, their colors in Lacy et al. (2004) color space tend to be quite blue (see Figure 12). The reason for this behavior is illustrated in Figure 14. While our new AGN selection criteria require sources to have monotonically rising IRAC SEDs, only $15 \%$ of the "Stern-only" sample display this behavior. Instead, their SEDs tend to rise between 3.6 and $4.5 \mu \mathrm{m}$, turn over between 4.5 and $5.8 \mu \mathrm{m}$, and then rise again toward long wavelengths. As shown in Figure 14, at the sample's median redshift of $z=1.5$, this behavior can be attributed to the 3.6 and $4.5 \mu \mathrm{m}$ bands sampling the rising side of the $1.6 \mu \mathrm{m}$ stellar bump, the $5.8 \mu \mathrm{m}$ band falling redward of the bump, and the $8.0 \mu \mathrm{m}$ band sampling the bright MIR emission from the $3.3 \mu \mathrm{m}$ PAH feature and warm star-formation-heated dust. Moderateredshift star-forming galaxies like the one plotted in Figure 14 (which were excluded from the original Stern et al. 2005 sample by their shallow flux limit of $S_{8 \mu \mathrm{m}} \geqslant 76 \mu \mathrm{Jy}$ ) will therefore mimic power-law AGNs when adjacent wavebands are used to determine their IRAC colors, but can effectively be separated from AGNs using a longer wavelength baseline or a requirement that the SED rise monotonically.

\section{SUMMARY}

IRAC selection provides a powerful tool for identifying luminous AGNs. However, the AGN selection wedges currently in use (e.g., Lacy et al. 2004; Stern et al. 2005) are heavily contaminated by normal star-forming galaxies in deep IRAC data. Using the large samples of luminous AGNs and highredshift star-forming galaxies in COSMOS, we redefine the IRAC AGN selection criteria for use in deep IRAC surveys. The new cuts, presented in Section 9, incorporate the best aspects of the current AGN selection wedges and of infrared power-law selection (Alonso-Herrero et al. 2006; Donley et al. 2007; Park et al. 2010), while improving on both. They are designed to be both highly complete and reliable, and effectively exclude highredshift star-forming galaxies selected via the BzK, DRG, LBG, and SMG criteria down to IRAC flux limits of $0.9,1.7,11.3$, and $14.6 \mu \mathrm{Jy}$ in the $3.6,4.5,5.8$, and $8.0 \mu \mathrm{m}$ bands, respectively.

The completeness of the new selection criteria is highly luminosity dependent. While fewer than $20 \%$ of Seyfert galaxies with $\log L_{2-10 \mathrm{keV}}\left(\mathrm{erg} \mathrm{s}^{-1}\right) \leqslant 43$ meet the IRAC selection criteria, the new cuts recover $75 \%$ of hard X-ray-detected AGNs with QSO-luminosities of $\log L_{2-10 \mathrm{kev}}\left(\mathrm{erg} \mathrm{s}^{-1}\right) \geqslant 44$. Despite this bias toward luminous AGNs, however, only $38 \%$ of the IRAC-selected AGN candidates in COSMOS would be identified as AGNs in the X-ray, a fraction that rises to $52 \%$ in regions with deep Chandra data $\left(T_{\mathrm{x}}=50-160 \mathrm{ks}\right)$.

$\mathrm{X}$-ray stacking of the individually X-ray-non-detected AGN candidates leads to $\geqslant 6 \sigma$ detections in both the hard and soft $\mathrm{X}$-ray bands, with a large implied column density of $\log$ $N_{\mathrm{H}}\left(\mathrm{cm}^{-2}\right) \sim 23.5 \pm 0.4$. In comparison, the hard X-rayselected AGNs have a typical column density of only log $N_{\mathrm{H}}\left(\mathrm{cm}^{-2}\right)=22.4 \pm 0.4$. While some X-ray-non-detected AGNs are likely to be missed in the X-ray because of their higher typical redshifts $(z \sim 2.2$ compared to $z \sim 1.7$ for the X-ray AGNs), heavily obscured to mildly Compton-thick obscuration appears to be primarily responsible for driving these intrinsically luminous AGNs below the current X-ray flux limits.

The selection criteria defined here provide a reliable method for identifying highly complete samples of luminous unobscured and obscured AGNs with high-quality (e.g., $\gtrsim 5 \sigma$ ) counterparts in the four IRAC bands, a condition met by $88 \%$ of the $X M M$-COSMOS sample. However, IRAC selection cannot effectively identify low-luminosity AGNs with host-dominated MIR SEDs, and also appears to be incomplete to luminous heavily obscured AGNs with particularly bright hosts (e.g., type 2 radio galaxies). X-ray and radio selection therefore remain important tools for identifying unobscured to moderately obscured low-luminosity AGNs and obscured radio-loud AGNs, respectively. While the upcoming $>10 \mathrm{keV}$ NuSTAR X-ray mission (Harrison et al. 2010) will begin to probe the population of heavily obscured Seyfert-luminosity AGNs at low to moderate redshift ( $z \lesssim 0.4$; e.g., Ballantyne et al. 2011), new methods such as composite SED fitting (e.g., A. Del Moro, in preparation) will be required to identify the distant radio-quiet and heavily obscured Seyfert-luminosity AGNs missed by current $\mathrm{X}$-ray, radio, and MIR-based selection techniques.

We thank Ranga Chary, Harry Ferguson, Joanna Kuraszkiewicz, Mark Lacy, Daniel Stern, Belinda Wilkes, Steve Willner, and the anonymous referee for helpful discussions and comments that improved this paper. J.L.D. is supported by the Giacconi Fellowship at STScI. This work is partially supported by CONACyT Apoyo 83564, DGAPA UNAM Grant PAPIIT IN110209, and NASA ADP Grant NNX07AT02G.

\section{APPENDIX}

At high redshift, the observed X-ray bands sample progressively harder X-ray emission less sensitive to intrinsic obscuration. As a result, low column densities become poorly constrained and are often overestimated (see also Ueda et al. 2003; Akylas et al. 2006). To illustrate and quantify this bias, 

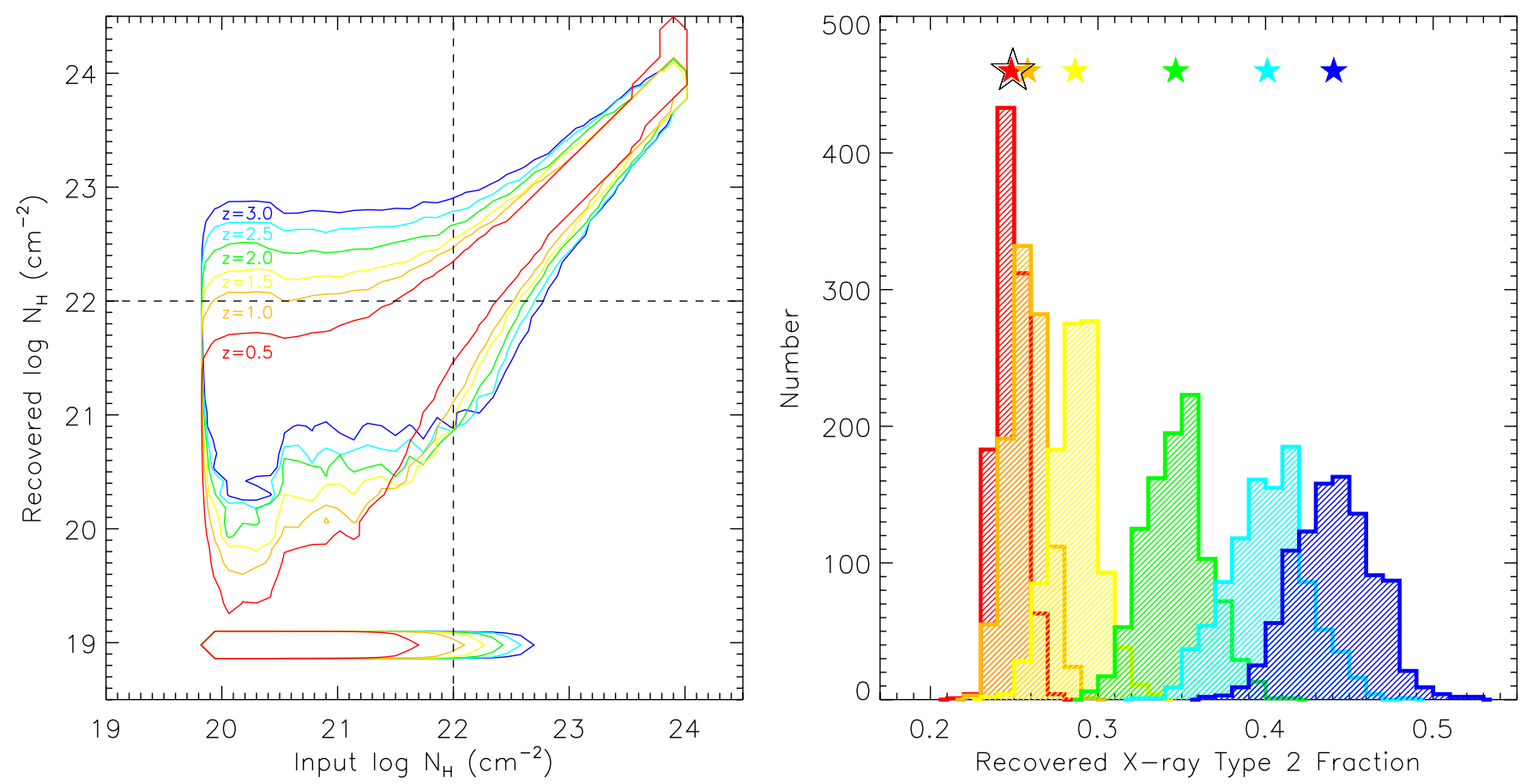

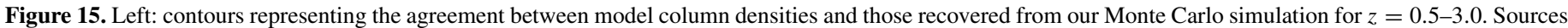

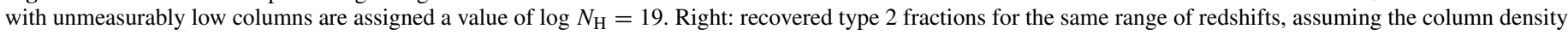

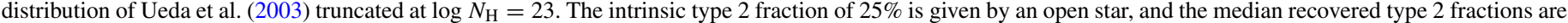

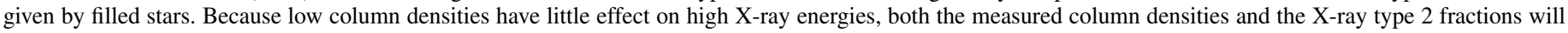
be overestimated at high redshift.

(A color version of this figure is available in the online journal.)

we use a Monte Carlo simulation to study the effect of observational errors on the measured column densities of X-raydetected AGNs (but do not consider here any additional biases in the type 2 fraction introduced by X-ray detectability as a function of redshift and/or obscuration).

We start with the intrinsic column density distribution of QSO-luminosity AGNs $\left(\log L_{\mathrm{x}}\left(\mathrm{erg} \mathrm{s}^{-1}\right) \sim 44\right)$ from Ueda et al. (2003), which we parameterize as 500 discrete columns. For redshifts of $z=0.5-3.0$, we then calculate the hard and soft $\mathrm{X}$-ray fluxes that would be observed for each of the input columns, and vary these ideal fluxes using random Gaussian errors scaled by the observed errors on the X-ray fluxes of the $X M M$-COSMOS sample. Using these randomized fluxes, we then remeasure the column density using the method described in Section 5.1. The results are shown in Figure 15(a). While low column densities are more poorly constrained than high column densities even at low redshift, very few unobscured AGNs $\left(\log N_{\mathrm{H}}\left(\mathrm{cm}^{-2}\right)<22\right)$ will be misclassified as obscured AGNs $\left(\log N_{\mathrm{H}}\left(\mathrm{cm}^{-2}\right) \geqslant 22\right)$ at $z \lesssim 0.5$. However, as the redshift increases, progressively larger fractions of intrinsically unobscured AGNs will scatter into the obscured region of Figure 15(a). Because heavily obscured AGNs are far less likely to be misclassified as unobscured, this observational scatter leads to an apparent increase in the type 2 fraction of highredshift X-ray-detected AGNs.

To estimate the effect of this bias on the measured X-ray type 2 fraction of the hard X-ray-detected $X M M$-COSMOS sample, $89 \%$ of which have $\log N_{\mathrm{H}}\left(\mathrm{cm}^{-2}\right) \leqslant 23$, we first truncate the Ueda et al. (2003) column density distribution at $\log N_{\mathrm{H}}\left(\mathrm{cm}^{-2}\right)$ $=23$. The resulting type 2 fractions recovered from the Monte Carlo simulation are shown in Figure 15(b), as is the intrinsic value of $25 \%$. For the assumed column density distribution, the recovered X-ray type 2 fraction is overestimated by only
$4 \%$ at $z=1$, the approximate redshift of the bluest XMM sources in Figure 7. However, at $z=2$, the typical redshift of the reddest $X M M$ sources, the observed type 2 fraction is likely to be overestimated by $\sim 40 \%$. This overestimation of the $\mathrm{X}$-ray type 2 fraction at high redshift contributes to the apparent discrepancies between X-ray- and optically classified AGNs (see Sections 5 and 6).

\section{REFERENCES}

Adelman-McCarthy, J. K., Agüeros, M. A., Allam, S. S., et al. 2006, ApJS, 162, 38

Akylas, A., Georgantopoulos, I., Georgakakis, A., Kitsionas, S., \& Hatziminaoglou, E. 2006, A\&A, 459, 693

Alonso-Herrero, A., Pérez-González, P. G., Alexander, D. M., et al. 2006, ApJ, 640, 167

Assef, R. J., Kochanek, C. S., Ashby, M. L. N., et al. 2011, ApJ, 728, 56

Assef, R. J., Kochanek, C. S., Brodwin, M., et al. 2010, ApJ, 713, 970

Ballantyne, D. R., Draper, A. R., Madsen, K. K., Rigby, J. R., \& Treister, E. 2011, ApJ, 736, 56

Ballantyne, D. R., Everett, J. E., \& Murray, N. 2006, ApJ, 639, 740

Barmby, P., Alonso-Herrero, A., Donley, J. L., et al. 2006, ApJ, 642, 126

Barmby, P., Huang, J., Ashby, M. L. N., et al. 2008, ApJS, 177, 431

Bouwens, R. J., Illingworth, G. D., Franx, M., et al. 2009, ApJ, 705, 936

Brand, K., Dey, A., Weedman, D., et al. 2006, ApJ, 644, 143

Brand, K., Weedman, D. W., Desai, V., et al. 2008, ApJ, 673, 119

Brusa, M., Civano, F., Comastri, A., et al. 2010, ApJ, 716, 348

Brusa, M., Fiore, F., Santini, P., et al. 2009, A\&A, 507, 1277

Buchanan, C. L., Gallimore, J. F., O'Dea, C. P., et al. 2006, AJ, 132, 401

Caccianiga, A., Severgnini, P., Della Ceca, R., et al. 2007, A\&A, 470, 557

Capak, P., Aussel, H., Ajiki, M., et al. 2007, ApJS, 172, 99

Capak, P., Carilli, C. L., Lee, N., et al. 2008, ApJ, 681, L53

Capak, P. L., Riechers, D., Scoville, N. Z., et al. 2011, Nature, 470, 233

Cappelluti, N., Brusa, M., Hasinger, G., et al. 2009, A\&A, 497, 635

Cardamone, C. N., Urry, C. M., Damen, M., et al. 2008, ApJ, 680, 130

Civano, F., Mignoli, M., Comastri, A., et al. 2007, A\&A, 476, 1223

Cleary, K., Lawrence, C. R., Marshall, J. A., Hao, L., \& Meier, D. 2007, ApJ, 660,117 
Comastri, A., Mignoli, M., Ciliegi, P., et al. 2002, ApJ, 571, 771

Conselice, C. J., Newman, J. A., Georgakakis, A., et al. 2007, ApJ, 660, L55

Coppin, K., Pope, A., Menéndez-Delmestre, K., et al. 2010, ApJ, 713, 503

Coppin, K. E. K., Smail, I., Alexander, D. M., et al. 2009, MNRAS, 395, 1905

Daddi, E., Alexander, D. M., Dickinson, M., et al. 2007a, ApJ, 670, 173

Daddi, E., Cimatti, A., Renzini, A., et al. 2004, ApJ, 617, 746

Daddi, E., Dannerbauer, H., Krips, M., et al. 2009a, ApJ, 695, L176

Daddi, E., Dickinson, M., Morrison, G., et al. 2007b, ApJ, 670, 156

Daddi, E., Dannerbauer, H., Stern, D., et al. 2009b, ApJ, 694, 1517

Dale, D. A., \& Helou, G. 2002, ApJ, 576, 159

Dasyra, K. M., Yan, L., Helou, G., et al. 2009, ApJ, 701, 1123

De Breuck, C., Seymour, N., Stern, D., et al. 2010, ApJ, 725, 36

Desai, V, Soifer, B. T., Dey, A., et al. 2009, ApJ, 700, 1190

Dey, A., Soifer, B. T., Desai, V., et al. 2008, ApJ, 677, 943

Donley, J. L., Rieke, G. H., Alexander, D. M., Egami, E., \& Pérez-González, P. G. 2010, ApJ, 719, 1393

Donley, J. L., Rieke, G. H., Pérez-González, P. G., \& Barro, G. 2008, ApJ, 687, 111

Donley, J. L., Rieke, G. H., Pérez-González, P. G., Rigby, J. R., \& AlonsoHerrero, A. 2007, ApJ, 660, 167

Draine, B. T. 2003, ARA\&A, 41, 241

Dunlop, J. S., Cirasuolo, M., \& McLure, R. J. 2007, MNRAS, 376, 1054

Eckart, M. E., McGreer, I. D., Stern, D., Harrison, F. A., \& Helfand, D. J. 2010, ApJ, 708, 584

Elvis, M., Civano, F., Vignali, C., et al. 2009, ApJS, 184, 158

Farrah, D., Lonsdale, C. J., Weedman, D. W., et al. 2008, ApJ, 677, 957

Farrah, D., Weedman, D., Lonsdale, C. J., et al. 2009, ApJ, 696, 2044

Fiore, F., Grazian, A., Santini, P., et al. 2008, ApJ, 672, 94

Fiore, F., Puccetti, S., Brusa, M., et al. 2009, ApJ, 693, 447

Franx, M., Labbé, I., Rudnick, G., et al. 2003, ApJ, 587, L79

Fu, H., Yan, L., Scoville, N. Z., et al. 2010, ApJ, 722, 653

Gandhi, P., Horst, H., Smette, A., et al. 2009, A\&A, 502, 457

Gawiser, E., van Dokkum, P. G., Herrera, D., et al. 2006, ApJS, 162, 1

Gilli, R., Comastri, A., \& Hasinger, G. 2007, A\&A, 463, 79

Gilli, R., Su, J., Norman, C., et al. 2011, ApJ, 730, L28

Haas, M., Willner, S. P., Heymann, F., et al. 2008, ApJ, 688, 122

Hao, H., Elvis, M., Civano, F., \& Lawrence, A. 2011, ApJ, 733, 108

Hao, H., Elvis, M., Civano, F., et al. 2010, ApJ, 724, L59

Harrison, F. A., Boggs, S., Christensen, F., et al. 2010, Proc. SPIE, 7732, 77320 Hasinger, G. 2008, A\&A, 490, 905

Hasinger, G., Cappelluti, N., Brunner, H., et al. 2007, ApJS, 172, 29

Heckman, T. M. 1995, ApJ, 446, 101

Hickox, R. C., Jones, C., Forman, W. R., et al. 2007, ApJ, 671, 1365

Hou, L. G., Han, J. L., Kong, M. Z., \& Wu, X.-B. 2011, ApJ, 732, 72

Houck, J. R., Weedman, D. W., Le Floc'h, E., \& Hao, L. 2007, ApJ, 671, 323

Huang, J., Faber, S. M., Daddi, E., et al. 2009, ApJ, 700, 183

Ilbert, O., Capak, P., Salvato, M., et al. 2009, ApJ, 690, 1236

Ilbert, O., Salvato, M., Le Floc'h, E., et al. 2010, ApJ, 709, 644

Kauffmann, G., Heckman, T. M., Tremonti, C., et al. 2003, MNRAS, 346, 1055

Knudsen, K. K., Kneib, J., Richard, J., Petitpas, G., \& Egami, E. 2010, ApJ, 709,210

Koekemoer, A. M., Aussel, H., Calzetti, D., et al. 2007, ApJS, 172, 196

Lacy, M., Bunker, A. J., \& Ridgway, S. E. 2000, AJ, 120, 68

Lacy, M., Petric, A. O., Sajina, A., et al. 2007, AJ, 133, 186

Lacy, M., Storrie-Lombardi, L. J., Sajina, A., et al. 2004, ApJS, 154, 166

Lane, K. P., Almaini, O., Foucaud, S., et al. 2007, MNRAS, 379, L25

Lehmer, B. D., Brandt, W. N., Alexander, D. M., et al. 2008, ApJ, 681, 1163

Leipski, C., Haas, M., Willner, S. P., et al. 2010, ApJ, 717, 766

Lilly, S. J., Le Fèvre, O., Renzini, A., et al. 2007, ApJS, 172, 70

Lusso, E., Comastri, A., Vignali, C., et al. 2010, A\&A, 512, A34

Lusso, E., Comastri, A., Vignali, C., et al. 2011, A\&A, 534, A110
Lutz, D., Maiolino, R., Spoon, H. W. W., \& Moorwood, A. F. M. 2004, A\&A, 418, 465

Mainieri, V., Hasinger, G., Cappelluti, N., et al. 2007, ApJS, 172, 368

Maiolino, R., Shemmer, O., Imanishi, M., et al. 2007, A\&A, 468, 979

Mancini, C., Matute, I., Cimatti, A., et al. 2009, A\&A, 500, 705

Marchesini, D., Whitaker, K. E., Brammer, G., et al. 2010, ApJ, 725, 1277

McCracken, H. J., Capak, P., Salvato, M., et al. 2010, ApJ, 708, 202

Menéndez-Delmestre, K., Blain, A. W., Smail, I., et al. 2009, ApJ, 699, 667

Moran, E. C., Filippenko, A. V., \& Chornock, R. 2002, ApJ, 579, L71

Nenkova, M., Sirocky, M. M., Ivezić, Ž., \& Elitzur, M. 2008a, ApJ, 685, 147

Nenkova, M., Sirocky, M. M., Nikutta, R., Ivezić, Ž., \& Elitzur, M. 2008b, ApJ, 685,160

Ogle, P., Whysong, D., \& Antonucci, R. 2006, ApJ, 647, 161

Page, M. J., Loaring, N. S., Dwelly, T., et al. 2006, MNRAS, 369, 156

Papovich, C., Cool, R., Eisenstein, D., et al. 2006, AJ, 132, 231

Park, S. Q., Barmby, P., Willner, S. P., et al. 2010, ApJ, 717, 1181

Pérez-González, P. G., Rieke, G. H., Villar, V., et al. 2008, ApJ, 675, 234

Pier, E. A., \& Krolik, J. H. 1993, ApJ, 418, 673

Polletta, M., Weedman, D., Hönig, S., et al. 2008, ApJ, 675, 960

Pope, A., Chary, R.-R., Alexander, D. M., et al. 2008, ApJ, 675, 1171

Prescott, M. K. M., Impey, C. D., Cool, R. J., \& Scoville, N. Z. 2006, ApJ, 644, 100

Reach, W. T., Megeath, S. T., Cohen, M., et al. 2005, PASP, 117, 978

Reddy, N. A., Erb, D. K., Steidel, C. C., et al. 2005, ApJ, 633, 748

Reddy, N. A., Steidel, C. C., Pettini, M., et al. 2008, ApJS, 175, 48

Richards, G. T., Deo, R. P., Lacy, M., et al. 2009, AJ, 137, 3884

Riechers, D. A., Capak, P. L., Carilli, C. L., et al. 2010, ApJ, 720, L131

Rieke, G. H., Alonso-Herrero, A., Weiner, B. J., et al. 2009, ApJ, 692, 556

Rodighiero, G., Cimatti, A., Franceschini, A., et al. 2007, A\&A, 470, 21

Sajina, A., Lacy, M., \& Scott, D. 2005, ApJ, 621, 256

Salvato, M., Hasinger, G., Ilbert, O., et al. 2009, ApJ, 690, 1250

Salvato, M., Ilbert, O., Hasinger, G., et al. 2011, ApJ, 742, 61

Sanders, D. B., Salvato, M., Aussel, H., et al. 2007, ApJS, 172, 86

Sargsyan, L. A., \& Weedman, D. W. 2009, ApJ, 701, 1398

Schinnerer, E., Carilli, C. L., Capak, P., et al. 2008, ApJ, 689, L5

Scoville, N., Aussel, H., Brusa, M., et al. 2007, ApJS, 172, 1

Seymour, N., Stern, D., De Breuck, C., et al. 2007, ApJS, 171, 353

Sharp, R. G., Sabbey, C. N., Vivas, A. K., et al. 2002, MNRAS, 337, 1153

Shim, H., Chary, R., Dickinson, M., et al. 2011, ApJ, 738, 69

Smolčić, V., Capak, P., Ilbert, O., et al. 2011, ApJ, 731, L27

Steidel, C. C., Adelberger, K. L., Shapley, A. E., et al. 2003, ApJ, 592, 728

Stern, D., Eisenhardt, P., Gorjian, V., et al. 2005, ApJ, 631, 163

Tozzi, P., Gilli, R., Mainieri, V., et al. 2006, A\&A, 451, 457

Treister, E., Urry, C. M., Chatzichristou, E., et al. 2004, ApJ, 616, 123

Treister, E., Urry, C. M., Van Duyne, J., et al. 2006, ApJ, 640, 603

Trouille, L., Barger, A. J., Cowie, L. L., Yang, Y., \& Mushotzky, R. F. 2009, ApJ, 703,2160

Trump, J. R., Impey, C. D., Elvis, M., et al. 2009, ApJ, 696, 1195

Trump, J. R., Impey, C. D., Kelly, B. C., et al. 2011a, ApJ, 733, 60

Trump, J. R., Impey, C. D., McCarthy, P. J., et al. 2007, ApJS, 172, 383

Trump, J. R., Nagao, T., Ikeda, H., et al. 2011b, ApJ, 732, 23

Ueda, Y., Akiyama, M., Ohta, K., \& Miyaji, T. 2003, ApJ, 598, 886

van Dokkum, P. G., Förster Schreiber, N. M., Franx, M., et al. 2003, ApJ, 587, L83

van Dokkum, P. G., Quadri, R., Marchesini, D., et al. 2006, ApJ, 638, L59

Weedman, D., Polletta, M., Lonsdale, C. J., et al. 2006, ApJ, 653, 101 Weedman, D. W., \& Houck, J. R. 2009, ApJ, 693, 370

Wiklind, T., Dickinson, M., Ferguson, H. C., et al. 2008, ApJ, 676, 781

Yan, H., Dickinson, M., Giavalisco, M., et al. 2006, ApJ, 651, 24

Yun, M. S., Aretxaga, I., Ashby, M. L. N., et al. 2008, MNRAS, 389, 333 